\title{
Spatiotemporal evolution of evaporating liquid films sheared by a gas
}

\author{
Omair A. A. Mohamed $\odot^{*}$ \\ Mechanical Engineering Department, Bilkent University, 06800 Ankara, Turkey \\ Michael C. Dallaston $\odot^{\dagger}$ \\ School of Mathematical Sciences, Queensland University of Technology, \\ Brisbane, Queensland 4000, Australia \\ Luca Biancofiore $0^{*}$ \\ Mechanical Engineering Department, Bilkent University, 06800 Ankara, Turkey
}

(Received 5 May 2021; accepted 11 October 2021; published 4 November 2021)

We study the spatiotemporal evolution of an evaporating liquid film sheared by a gas considering both inertial and thermal instabilities, the latter arising from a combination of evaporation and Marangoni effects. The shearing gas is modeled by imposing a constant shear stress applied along the liquid's interface. Following in the footsteps of Joo et al. [S. W. Joo et al., J. Fluid Mech. 230, 117 (1991)], long-wave theory is used to derive a Benney-like equation governing the temporal evolution of the liquid interface under the effects of inertia, hydrostatic pressure, surface tension, thermocapillarity, evaporation, and gas shear. Linear stability theory is used to investigate the temporal and spatiotemporal characteristics of the flow, where it is found that the evaporation of the film promotes absolute instabilities and can cause convective-absolute transitions of the perturbations. It is also found that a strong enough counterflowing shearing gas can suppress the inertial instability, commonly known as the $H$ mode, affirming similar conclusions found by previous studies for a strongly confined isothermal film. Additionally, our temporal stability analysis indicates that the thinning of the film reduces the phase speed of thermal perturbations, due to the increasing dominance of viscosity. However, our spatiotemporal analysis shows that the thinning of the film actually results in the growth of additional modes with higher group velocities resulting in faster contamination of the flow field. Moreover, the interface evolution equation is solved numerically to (i) simulate the film's interface evolution subject to finite perturbations and (ii) compare to the results of the linear stability analysis. We find qualitative agreement between the temporal dynamics of the linear and nonlinear instabilities. Our subsequent numerical nonlinear spatiotemporal stability analysis demonstrates that for weaker thermal instabilities, the wave-front dynamics are imposed by the nonlinearly saturated wave packet, while for stronger thermal instabilities, the wave-front dynamics are dictated by the linear dispersion relationship. We also study the effects of the dimensionless parameters on the rupture location and the time it takes for the fluid film to rupture. Finally, the shear stress's effect on the rupture mechanics of the film is studied using self-similarity analysis, where we identify the fate of the evolution equation's solutions.

\footnotetext{
*omair.mohamed@bilkent.edu.tr

†michael.dallaston@qut.edu.au

Corresponding author: luca@bilkent.edu.tr
} 
DOI: 10.1103/PhysRevFluids.6.114002

\section{INTRODUCTION}

Liquid films are utilized across a wide range of technologies, including rocket engine nozzle protection, the cooling of electronics, coating processes, and postcombustion carbon dioxide capture. In numerous applications the liquid film is volatile and subject to a shearing gas such as in distillation columns [1], structured packings [2], gas scrubbers [3], and airblast atomizers [4]. The spatiotemporal evolution of the liquid films' interfaces is tied directly to the performance and efficiency of the systems they are in; for instance, a shear instability akin to the Kelvin-Helmholtz type has been shown to be the mechanism by which the fluid interface is initially destabilized in airblast atomization devices [5]. Consequently, the evolution of a liquid film's interface has garnered significant attention, particularly investigating the domains for which it is stable or unstable to disturbances by means of stability theory. The importance of these studies is further enhanced by the fact that they can shed light on the origins of turbulence in liquid films since some finite turbulent structures can be traced back to specific linear instabilities [6].

The dynamics of thin liquid films has been studied extensively since the early pioneering theoretical and experimental work of Kapitza [7,8], who first identified the hydrodynamic instability mode known as the Kapitza mode or $H$-mode instability. These early works on isothermal films were followed by studies conducted by Benjamin [9] and Yih [10], who proved that a vertical liquid film is always unstable to long-wave perturbations and identified the critical Reynolds number at the onset of the instability. Benney [11] used a long-wave expansion to formulate a temporal evolution equation accounting for the different forces governing the flow. The validity of this long-wave approximation was significantly extended by Ruyer-Quil and Manneville [12,13] by integrating a systematic gradient expansion with weighted residual techniques. Notably, Kapitza's explanation of the instability mechanism to be driven by the balance between viscous dissipation and the work of gravity was erroneous and was corrected by Smith [14], who determined the instability mechanism to be driven by inertia. Further arguments regarding inertia's role were made by Kalliadasis et al. [15] and Dietze [16].

Thermocapillary forces along with gravity were first considered by Sreenivasan and Lin [17] in a linear stability study on a film flowing down an inclined surface held at a constant temperature. They found that including the stabilizing effects of gravity resulted in a nonzero critical Marangoni number. Kelly et al. [18] later applied long-wave theory to find two critical Reynolds numbers associated with the thermocapillary instability and the modified $H$-mode instability. This thermocapillary instability was later classified into two distinct modes by Goussis and Kelly [19]. They identified (i) the long-wave $S$-mode instability driven by changes in surface tension across the interface and (ii) the short-wave $P$-mode instability driven by the interaction between the perturbation velocity and the base state temperature gradient.

The study of the stability of fluid films was also expanded to include the effects of phase change. Under some of the models developed, the evaporation of the liquid film is limited by the molecular transfer rate, as determined by the divergence from thermodynamic equilibrium at the interface. Studies focusing on this limiting case include that of Burelbach et al. [20], who investigated the stability of evaporating and condensing films by formulating a one-sided evaporation model decoupling the liquid and vapor dynamics and subsequently utilized long-wave theory to study the effects of evaporation and condensation on the stability of the liquid film. This model was extended by Joo et al. [21], who considered a flowing film subject to gravitational effects, but neglected disjoining pressure forces. Both of these works found evaporation to be a destabilizing effect. Recently, Mohamed and Biancofiore [22] derived the Orr-Sommerfeld system from the one-sided model of Burelbach et al. [20] to investigate the evaporation-induced instability in falling liquid films without the limitations of long-wave theory. They referred to the evaporative instability as the E-mode; we use the terminology $E$ mode herein. Other sophisticated one-sided evaporation models focused on accounting for the interaction between evaporation effects and disjoining pressure forces, 
such as those developed by Ajaev and Homsy [23-25]. Following a similar approach, the contest between the effects of weak phase change and dewetting in thin liquid films was examined by Ji and Witelski [26], who observed the emergence of rich transient behavior encompassing the dynamics of both uniform films and droplets. Other notable contributions to the stability of volatile films include the work of Howison et al. [27], who investigated the problem of a nonhomogeneous fluid consisting of a volatile solvent and a nonvolatile resin, e.g., a drying paint layer. They derived a governing system of equations which accounted for variable solvent evaporation rates and solvent diffusivity and which captured flow reversals and the appearance of perturbations in the flat layer due to concentration gradients. Furthermore, Shklyaev and Fried [28] used a newly developed set of interface boundary conditions [29] based on the balance of configurational momentum instead of the conventional Hertz-Knudsen-Langmuir equation. On the other hand, the evaporation of the liquid film can be limited by the diffusion rate into the neighboring phase due to the saturation of the layer of gas immediately above the liquid interface, especially where there is no gas convection above the liquid [30-32]. Sultan et al. [32,33] derived a general model accounting for both transfer-ratelimited and diffusion-limited evaporation. Notably, they found evaporation in the diffusion-limited case to actually be a stabilizing effect.

Accounting for the presence of a gas layer adjacent to the liquid film greatly increases the complexity of the problem and due to its significant practical applications has been a subject of particular interest. Early experimental works made in this regard include the works of Hanratty and Engen [34], who demonstrated the various wave transitions in air-water flows, and Craik [35], who detected waves which travel faster than the interface and others that travel slower than it in film flows inside horizontal channels. More recently, O’Náraigh et al. [36] formulated a single equation accounting for interfacial turbulence and found that the wave-induced Reynolds stresses do not play an important role in the growth of interfacial waves. Dietze and Ruyer-Quil [37] formulated a low-dimensional model based on a weighted-residual integral boundary layer approach to study the interaction between the gas and liquid phases for horizontal pressure-driven and gravity-driven liquid films, in the cases of both co- and counterflowing gas phases. Their model successfully predicted the instability onset, phase speed, and growth rate in the long-wave limit. Lavalle et al. [38] found that the effect of the counterflowing gas can be stabilizing or destabilizing or cause harmonic changes in the cutoff wave number and temporal growth rate depending on the level of confinement of the liquid film. Notably, they found that the $H$-mode instability can be completely suppressed by a counterflowing gas given sufficient confinement. Due to the complexity of the coupled gas-liquid problem, a common approach is to simplify the problem by imposing the gas stress at the liquid-gas interface and solving for the liquid phase only. King et al. [39] considered a thin isothermal film flowing down an inclined plane subject to a counterflowing steam of air. The shear stress applied by the airflow onto the liquid interface was prescribed via a constant drag coefficient, while its normal stress was variable and related to the constant shear stress by thin-airfoil theory. Moreover, Sivapuratharasu et al. [40] considered the same problem and in similar fashion assumed the airflow's shear stress to be constant along the liquid interface but neglected its contribution to the normal stress balance.

Another important phenomenon associated with countercurrent gas flow over a liquid film is flooding, which refers to the appearance of large-amplitude standing waves in the fluid film at a critical shearing gas flow rate [41,42]. Additional increases in the gas flow rate beyond the flooding limit lead to the instabilities traveling in the upstream direction and possibly the complete reversal of the liquid flow direction, which can have severe consequences on the performance of systems such as plate heat exchangers, reflux condensers, and nuclear reactor cooling systems. This sudden onset of flooding is associated with a transition in the flow dynamics from convectively unstable to absolutely unstable [43].

The distinction between convective and absolute instabilities was first made by Briggs [44] in the field of plasma physics and later introduced into fluid stability theory by Huerre and Monkewitz [45]. In this framework, flows are classified by examining their long-time response to an infinitesimal impulse source. If the disturbance grows but is convected away by the flow, 
then the flow is classified as convectively unstable. On the other hand, if it grows at its origin and proceeds to contaminate the entire flow field, then the flow is classified as absolutely unstable. The spatiotemporal characterization of a falling liquid film was first performed by Brevdo et al. [46], who, by solving the Orr-Sommerfeld eigenvalue problem, found the instabilities to be of the convective type, a result identical to that found by Joo and Davis [47], who had used a model based on Benney's long-wave approach. O'Náraigh et al. [48] employed Orr-Sommerfeld stability theory along with a combination of linear modal analysis and ray analysis to study the problem of two-phase stratified flow of a laminar liquid phase and a fully developed gas phase which can be either turbulent or laminar. Using this model, they identified the regimes that produce absolute instabilities and compared their results to experimental flow-regime maps. In order to investigate the flooding phenomenon, Tseluiko and Kalliadasis [49] treated the liquid interface as a solid wall which permitted obtaining closed-form solutions for the pressure and tangential stresses imposed by the gas. The same model was applied by Vellingiri et al. [43] to investigate the linear spatiotemporal stability of an isothermal liquid film flowing down an inclined plane and subject to a counterflowing turbulent gas. They proceeded to identify the relationship between the gas flow rate and inclination angle, and the convective-absolute transitions in the flow's stability character. Additionally, they conceived a generalized methodology for identifying the type of instability when the flow is described by nonlocal equations.

A notable property of thin liquid films has been their tendency to spontaneously rupture once the film thickness reaches a critical value depending on the different forces competing within the film, such as disjoining pressure and surface tension [50]. Burelbach et al. [20] and Joo et al. [21] predicted the occurrence of film rupture due to long-wave instabilities driven by vapor recoil and thermocapillarity. The occurrence of film rupture was confirmed by the direct numerical simulations of nonvolatile films performed by Krishnamoorthy et al. [51], whose results showed good qualitative agreement with those of long-wave theory. Boos and Thess [52] demonstrated that the rupture caused by the long-wave Marangoni instability occurs via an an infinite cascade of increasingly smaller structures. Shklyaev et al. [53] considered a generalized one-dimensional Cahn-Hilliard equation suited for modeling the dynamics of a thin liquid film heated from below and subject to the Marangoni instability. They constructed self-similar solutions corresponding to the film dynamics and defined a power law governing their evolution. Wei and Duan [54] considered a liquid film undergoing quasiequilibrium evaporation and identified a set of self-similar solutions along with the power laws governing their behavior. Dallaston et al. [55] demonstrated that the formation of iterated structures as the film ruptures is due to a bifurcation of the continuous self-similar solution set into a discrete one occurring periodically over a logarithmic timescale. Another recent effort is that of Ji and Witelski [56], who investigated the evaporation-driven rupture of thin films using an elaborate evaporation model, which they used to examine the balance between conservative restoring forces and the nonconservative flux term. They found several categories of finite-time singularities including self-similar and non-self-similar behavior, which were studied both analytically and numerically.

In this work we consider an evaporating liquid film flowing down an inclined plane driven by gravity while being sheared by a gas. We prescribe the shear stress imparted on the free interface by the gas through the introduction of a constant value into the interface's shear stress balance and we neglect its normal stress since we assume it to be much less significant than the shear stress at the leading order [39]. In this model, the role of the shearing gas is confined to modifying the fluid base flow. Investigations of the effect of a shearing gas on thermal instabilities, i.e., those driven by thermocapillary and evaporation forces, are lacking. Therefore, our goal is to fill this gap. We also investigate the effects of surface shear on the self-similar solutions of the evolution equation close to film rupture.

Our paper is structured as follows. In Sec. II we introduce the governing equations, dimensionless parameters, and the modeling of the shear stress. We also derive the nonlinear governing equation using the same methodology employed by Benney [11] and Joo et al. [21]. We then use long-wave theory to derive an analytical dispersion relationship governing the dynamics of the film flow. In 


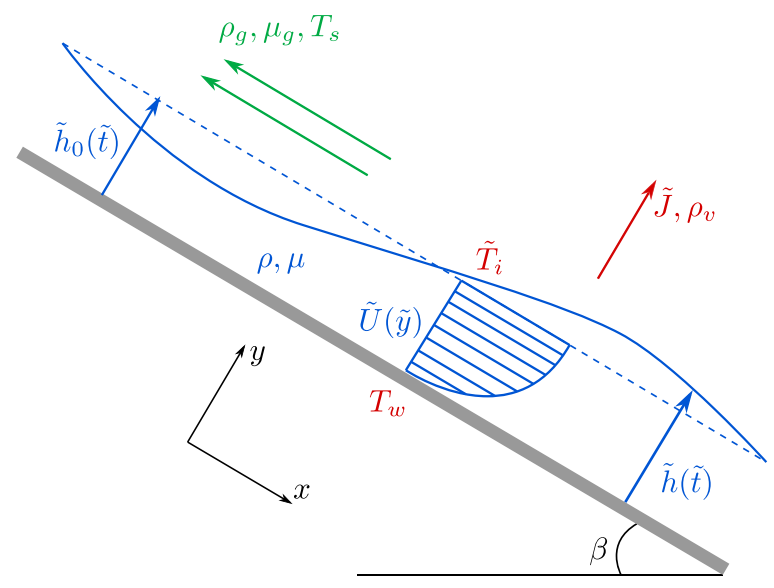

FIG. 1. Schematic illustration of a heated evaporating liquid film flowing under the influence of gravity while being sheared by a counterflowing gas. The dashed line represents the unperturbed base flow thickness $\tilde{h}_{0}, \beta$ is the inclination angle from the horizontal, and $T_{w}$ is the constant wall temperature.

Sec. III we perform a linear temporal stability analysis to study the effects of the shear stress on the stability of the flow, which is subsequently extended into a linear local spatiotemporal stability analysis. We further investigate the influence of the film thinning on the stability of the flow and how it is tied to the shear stress's role specifically. In Sec. IV we study the nonlinear dynamics of the flow by solving the governing equation numerically and we compare the temporal and spatiotemporal dynamics to the results of the linear stability analysis. We also study the influence of the dimensionless parameters on film rupture location and the time it takes for this rupture to occur. Moreover, we further investigate the film rupture dynamics via a self-similarity analysis and analyze the role of different dimensionless parameters on the rupture mechanism. A summary and our conclusions are presented in Sec. V.

\section{PROBLEM FORMULATION}

The problem we investigate is an extension of the studies of Burelbach et al. [20] and Joo et al. [21] to include the effects of a shearing gas. We consider an incompressible, thin, Newtonian, evaporating liquid film with constant physical properties, driven by gravity down a heated plate held at a constant temperature. The heated plate is inclined from the horizontal with an angle $\beta$ as depicted in Fig. 1. A Cartesian $(\tilde{x}, \tilde{y})$ coordinate system is considered where the $\tilde{x}$ coordinate points in the streamwise direction and the $\tilde{y}$ coordinate in a direction perpendicular to the heated plate, i.e., the cross-stream direction. The liquid film's interface is defined by the function $\tilde{y}=\tilde{h}(\tilde{x}, \tilde{t})$. Note that the tilde notation indicates dimensional quantities. The liquid film is sheared at its interface by a turbulent gas, whose effects are accounted for by a constant quantity denoted by $\tilde{\tau}$. This variable represents a constant shear stress applied along the entire interface and is derived in detail in Sec. II B. The liquid film is subject to two instability modes depending on the value of the flow's Reynolds number; for relatively small Reynolds numbers we consider a thermal instability mode resulting from the combination of the thermocapillary $S$-mode and the evaporative $E$-mode [22] instabilities. At larger Reynolds numbers the film is destabilized by the $H$-mode instability, modified by thermocapillarity and evaporation. 


\section{A. Governing equations}

The liquid film is governed by the continuity equation, Navier-Stokes momentum equations, and the energy equation, which in vector form read

$$
\begin{aligned}
\nabla \cdot \tilde{\mathbf{u}} & =0 \\
\partial_{\tilde{t}} \tilde{\mathbf{u}}+\tilde{\mathbf{u}} \cdot \nabla \tilde{\mathbf{u}} & =-\frac{1}{\rho} \nabla \tilde{p}+\nu \nabla^{2} \tilde{\mathbf{u}}+\tilde{\mathbf{F}} \\
\partial_{\tilde{t}} \tilde{T}+\tilde{\mathbf{u}} \cdot \nabla \tilde{T} & =\chi \nabla^{2} \tilde{T}
\end{aligned}
$$

where $\tilde{\mathbf{u}}=(\tilde{u}, \tilde{v})$ is the liquid velocity vector, $\rho$ is the liquid density, $\tilde{p}$ is the liquid's gauge pressure, $v$ is the liquid's kinematic viscosity, $\tilde{\mathbf{F}}=(g \sin \beta,-g \cos \beta)$ is the gravitational body force vector with $g$ the gravitational acceleration magnitude, $\tilde{T}$ is the liquid temperature, and $\chi=\lambda / \rho c_{p}$ is the liquid's thermal diffusivity such that $\lambda$ and $c_{p}$ are the thermal conductivity and constant pressure heat capacity, respectively. The boundary conditions at the solid wall $(\tilde{y}=0)$ are the no-slip, nopenetration condition on the velocity and the constant wall temperature:

$$
\begin{gathered}
\tilde{\mathbf{u}}=0, \\
\tilde{T}=T_{w} .
\end{gathered}
$$

Our evaporation model is that of Burelbach et al. [20], which is in turn based on the interface jump conditions derived by Delhaye [57], combined with a constitutive relationship based on kinetic theory [58]. The presence of gas flow above the liquid interface prevents the saturation of the gas phase with the liquid's vapor, and hence the evaporation process is transfer-rate-limited, as opposed to diffusion-limited evaporation, which would be expected in the case of a stationary fluid [30-32]. Notably, this model can be recovered from the general evaporation model of Sultan et al. [33] when a limit of no vapor diffusion is considered. Moreover, it has been demonstrated that the heat transfer from an evaporating liquid film subjected to laminar or turbulent gas flow is dominated by latent heat transport associated with the evaporation of the film, as opposed to sensible heat transfer, particularly heat convection due to the gas motion $[59,60]$. Therefore, we consider the effect of heat convection on the evaporation process to be negligible. The boundary conditions at the liquid-gas interface $\tilde{y}=\tilde{h}(\tilde{x}, \tilde{t}) \operatorname{read}$

$$
\begin{aligned}
\tilde{J}=\rho\left(\tilde{\mathbf{u}}-\tilde{\mathbf{u}}_{i}\right) \cdot \mathbf{n} & =\rho_{v}\left(\tilde{\mathbf{u}}_{v}-\tilde{\mathbf{u}}_{i}\right) \cdot \mathbf{n}, \\
\tilde{J}\left[\mathcal{L}+\frac{1}{2}\left(\frac{\tilde{J}}{\rho_{v}}\right)^{2}\right] & =-\lambda \nabla \tilde{T} \cdot \mathbf{n}, \\
\frac{\tilde{J}^{2}}{\rho_{v}} \mathbf{n}+\left(\tilde{\mathbf{T}}-\tilde{\mathbf{T}}_{g}\right) \cdot \mathbf{n} & =2 \tilde{\sigma} \kappa \mathbf{n}+\nabla_{s} \tilde{\sigma}
\end{aligned}
$$

Quantities with the subscript $i$ are at the liquid-gas interface, those with the subscript $v$ are vapor quantities, and those with the subscript $g$ are associated with the shearing gas. Here $\tilde{J}$ is the evaporation mass flux, $\mathcal{L}$ is the latent heat of vaporization, $\nabla_{s}$ is the surface gradient operator, $\kappa=-\frac{1}{2} \nabla_{s} \cdot \mathbf{n}$ is the mean interface curvature, and $\mathbf{n}$ is the local interface normal unit vector with modulus $N$ such that $\mathbf{n}=\frac{1}{N}\left(-\partial_{\tilde{x}} \tilde{h}, 1\right)$. Additionally, $\tilde{\sigma}=\sigma_{0}-\gamma\left(\tilde{T}_{i}-T_{s}\right)$ is the surface tension function considering $\sigma_{0}$ to be the mean surface tension at the saturation temperature $T_{s}$, while $\gamma=-d \tilde{\sigma} / d \tilde{T}$. Further, $\tilde{\mathbf{T}}=-\tilde{p}+\mathbf{I} \tilde{\mathbf{P}}$ is the general stress tensor consisting of the sum of the normal stress components and the deviatoric stress tensor $\tilde{\mathbf{P}}$. Finally, we utilize the constitutive relationship derived by Palmer [58], which reads

$$
\tilde{J}=\left(\frac{\alpha \rho_{v} \mathcal{L}}{T_{s}^{3 / 2}}\right)\left(\frac{M_{w}}{2 \pi R_{g}}\right)^{1 / 2}\left(\tilde{T}_{i}-T_{s}\right),
$$


where $\alpha$ is the accommodation coefficient, $M_{w}$ is the liquid's molecular weight, and $R_{g}$ is the universal gas constant. Other forces such as van der Waals are not considered in our model.

\section{B. Shear stress modeling}

We account for the gas's shear stress at the interface by first expanding $\tilde{\mathbf{T}}$ and $\tilde{\mathbf{T}}_{g}$ in Eq. (1h) to get

$$
\frac{\tilde{J}^{2}}{\rho_{v}} \mathbf{n}+\tilde{\mathbf{P}} \cdot \mathbf{n}-\tilde{p} \mathbf{n}-\tilde{\mathbf{P}}_{g} \cdot \mathbf{n}+\tilde{p}_{g} \mathbf{n}=2 \tilde{\sigma} \kappa \mathbf{n}+\nabla_{s} \tilde{\sigma}
$$

taking the dot product with $\mathbf{n}$ for the normal stress balance

$$
\frac{\tilde{J}^{2}}{\rho_{v}}+(\tilde{\mathbf{P}} \cdot \mathbf{n}) \cdot \mathbf{n}-\tilde{p}-\left(\tilde{\mathbf{P}}_{g} \cdot \mathbf{n}\right) \cdot \mathbf{n}+\tilde{p}_{g}=2 \tilde{\sigma} \kappa
$$

and taking the dot product with the tangent unit vector $\mathbf{t}=\frac{1}{N}\left(1, \partial_{\tilde{x}} \tilde{h}\right)$ for the shear stress balance in the $(\tilde{x}, \tilde{y})$ plane

$$
(\tilde{\mathbf{P}} \cdot \mathbf{n}) \cdot \mathbf{t}-\left(\tilde{\mathbf{P}}_{g} \cdot \mathbf{n}\right) \cdot \mathbf{t}=\nabla_{s} \tilde{\sigma} \cdot \mathbf{t}
$$

The quantity $-\left(\tilde{\mathbf{P}}_{g} \cdot \mathbf{n}\right) \cdot \mathbf{n}+\tilde{p}_{g}$ in Eq. (2b) is associated with the gas's normal stress and is neglected, while the quantity $\left(\tilde{\mathbf{P}}_{g} \cdot \mathbf{n}\right) \cdot \mathbf{t}$ in Eq. (2c) is replaced with a constant quantity denoted by $\tilde{\tau}$, whose magnitude represents the strength of the shear gas flowing against the interface. This is justified by the fact that under the long-wave expansion, the fluid layer's disruption of the gas flow is assumed to be minimal due to the fluid interface's slow variation in space; therefore, when the normal and shear stresses imparted by the gas on the interface are subjected to small perturbations, the leading-order normal stress is insignificant, while the leading-order shear stress effect is found to be quite substantial [39]. Under these assumptions, the role of the shearing gas is confined to modifying the fluid base flow. This modeling approach has been previously applied in a variety of fluid problems including droplets [61,62], rivulets [63-66], and thin liquid films [39,40]. Positive values of $\tilde{\tau}$ are indicative of a gas which is adding kinetic energy to the liquid interface by flowing along it in the same direction with higher momentum, while negative values of $\tilde{\tau}$ represent either a counterflowing gas or a coflowing gas which is taking away energy from the liquid flow by flowing along it with lower momentum.

\section{Scaling and dimensionless parameters}

The following scales are introduced into the system of equations (1) (see also Table I):

$$
\begin{aligned}
& (\tilde{x}, \tilde{y}, \tilde{z}) \rightarrow \tilde{h}_{0}\left(x^{*}, y^{*}, z^{*}\right), \quad(\tilde{u}, \tilde{v}, \tilde{w}) \rightarrow \frac{v}{\tilde{h}_{0}}\left(u^{*}, v^{*}, w^{*}\right), \quad \tilde{t} \rightarrow \frac{\tilde{h}_{0}^{2}}{v} t^{*}, \quad \tilde{h} \rightarrow \tilde{h}_{0} h^{*}, \\
& \tilde{T} \rightarrow T_{s}+T^{*} \Delta T, \quad \tilde{p} \rightarrow \frac{\rho v^{2}}{\tilde{h}_{0}^{2}} p^{*}, \quad \tilde{J} \rightarrow \frac{\lambda \Delta T}{\tilde{h}_{0} \mathcal{L}} J^{*} .
\end{aligned}
$$

Here $\Delta T=T_{w}-T_{s}, \tilde{h}_{0}$ is the mean film thickness at the initial time and the asterisk denotes nondimensional variables. Introducing these scales into Eqs. (1), in component form they 
TABLE I. Set of dimensionless parameters resulting from the scaling of Eqs. (1).

\begin{tabular}{lcl}
\hline \hline Parameter & Expression & \multicolumn{1}{c}{ Physical significance } \\
\hline Reynolds number Re & $g \tilde{h}_{0}^{3} / v^{2}$ & Ratio of inertial to viscous forces \\
Prandtl number Pr & $v / \chi$ & Ratio of momentum to thermal diffusivity \\
Evaporation number $E$ & $\lambda \Delta T / \rho \mathcal{L} v$ & Ratio of viscous to evaporative timescales \\
Density ratio $D$ & $3 \rho_{v} / 2 \rho$ & Ratio of vapor density to liquid density \\
Nondimensional latent heat $L$ & $8 \tilde{h}_{0}^{2} \mathcal{L} / 9 v^{2}$ & Measure of the liquid's latent heat \\
Nondimensional surface tension $S$ & $\sigma_{0} \tilde{h}_{0} / 3 \rho v^{2}$ & Measure of the liquid's surface tension \\
Capillary number Ca & $\gamma \Delta T / \sigma_{0}$ & Ratio of viscous to surface tension forces \\
Marangoni number Ma & $\gamma \Delta T \tilde{h}_{0} / 2 \mu \chi$ & Ratio of Marangoni to viscous forces \\
Dimensionless shear stress $\tau^{*}$ & $\left(N \tilde{h}_{0}^{2} / \mu \nu\right) \tilde{\tau}$ & Strength and direction of the shearing gas \\
Equilibrium parameter $K$ & $\frac{k T_{s}^{3 / 2} \sqrt{2 \pi R_{g}}}{\alpha \tilde{h}_{0} \rho_{v} \mathcal{L}^{2} \sqrt{\mathrm{M}_{w}}}$ & Degree of nonequilibrium at the liquid interface \\
\hline \hline
\end{tabular}

read

$$
\begin{aligned}
\partial_{x^{*}} u^{*}+\partial_{y^{*}} v^{*} & =0, \\
\partial_{t^{*}} u^{*}+u^{*} \partial_{x^{*}} u^{*}+v^{*} \partial_{y^{*}} u^{*} & =-\partial_{x^{*}} p^{*}+\partial_{x^{*} x^{*}} u^{*}+\partial_{y^{*} y^{*}} u^{*}+\operatorname{Re} \sin \beta, \\
\partial_{t^{*}} v^{*}+u^{*} \partial_{x^{*}} v^{*}+v^{*} \partial_{y^{*}} v^{*} & =-\partial_{y^{*}} p^{*}+\partial_{x^{*} x^{*}} v^{*}+\partial_{y^{*} y^{*}} v^{*}-\operatorname{Re} \cos \beta, \\
\operatorname{Pr}\left[\partial_{t^{*}} T^{*}+u^{*} \partial_{x^{*}} T^{*}+v^{*} \partial_{y^{*}} T^{*}\right] & =\partial_{x^{*} x^{*}} T^{*}+\partial_{y^{*} y^{*}} T^{*} .
\end{aligned}
$$

The scaled wall-boundary conditions at $\left(y^{*}=0\right)$ are

$$
u^{*}=v^{*}=0, \quad T^{*}=1
$$

while the scaled interface-boundary conditions at $y^{*}=h^{*}$ become

$$
\begin{gathered}
E J^{*}=\frac{1}{N}\left[-u^{*} \partial_{x^{*}} h^{*}+v^{*}-\partial_{t^{*}} h^{*}\right], \\
J^{*}+\frac{E^{2}}{D^{2} L} J^{* 3}=\frac{1}{N}\left[\partial_{x^{*}} h^{*} \partial_{x^{*}} T^{*}-\partial_{y^{*}} T^{*}\right] \\
-\frac{3}{2} \frac{E^{2}}{D} J^{* 2}-\frac{2}{N^{2}}\left[\partial_{x^{*}} u^{*}\left[\left(\partial_{x^{*}} h^{*}\right)^{2}-1\right]-\partial_{x^{*}} h^{*}\left(\partial_{y^{*}} u^{*}+\partial_{x^{*}} v^{*}\right)\right]+p^{*}=-3 S(1-C T)\left[\frac{\partial_{x^{*} x^{*}} h^{*}}{N^{3}}\right], \\
{\left[1-\left(\partial_{x^{*}} h^{*}\right)^{2}\right]\left[\partial_{y^{*}} u^{*}+\partial_{x^{*}} v^{*}\right]-4 \partial_{x^{*}} h^{*} \partial_{x^{*}} u^{*}-\tau^{*}=-2 \frac{M}{\operatorname{Pr}}\left[\partial_{x^{*}} T^{*}+\partial_{x^{*}} h^{*} \partial_{y^{*}} T^{*}\right] N} \\
K J^{*}=T^{*}
\end{gathered}
$$

\section{Long-wave theory and interface evolution equation}

In a procedure inspired by Benney's [11] expansion for small wave numbers, a small parameter $\epsilon=\frac{h^{*}}{\ell}$ is defined where $\ell$ is the flow's horizontal characteristic length such that $h^{*} \ll \ell$. Here $\epsilon$ is used to rescale the system's independent variables as

$$
x^{*} \rightarrow \epsilon x, \quad y^{*} \rightarrow y, \quad t^{*} \rightarrow \epsilon t .
$$


The star notation for $h^{*}$ and $\tau^{*}$ is be dropped from this point onward for convenience. Now the system's dependent variables are expanded in terms of $\epsilon$ as.

$$
\begin{aligned}
u & =u_{0}+\epsilon u_{1}+O\left(\epsilon^{2}\right), \\
v & =\epsilon\left[v_{0}+\epsilon v_{1}+O\left(\epsilon^{2}\right)\right], \\
p & =p_{0}+\epsilon p_{1}+O\left(\epsilon^{2}\right), \\
T & =T_{0}+\epsilon T_{1}+O\left(\epsilon^{2}\right), \\
J & =J_{0}+\epsilon J_{1}+O\left(\epsilon^{2}\right) .
\end{aligned}
$$

Additionally, the following dimensionless parameters are rescaled:

$$
E \rightarrow \epsilon \bar{E}, \quad D \rightarrow \epsilon^{2} \bar{D}, \quad S \rightarrow \frac{\bar{S}}{\epsilon^{2}}
$$

We consider the shear stress $\tau$ and the interface height function $h(t)$ to be zeroth-order quantities. Note that the transformations in (6) can be considered realistic when compared to the data tabulated in Ref. [20].

Substituting from Eqs. (4)-(6) into the system of equations (3), we obtain solutions for the dependent variables $(u, v, p, T, J)$ which can be substituted into Eq. (3f) to obtain

$$
\begin{aligned}
\partial_{t} h & +\partial_{x}\left[\frac{\operatorname{Re} \sin \beta}{3} h^{3}+\frac{\tau}{2} h^{2}\right]+\frac{\bar{E}}{h+K}+\epsilon \partial_{x}\left[\frac{2}{15}(\operatorname{Re} \sin \beta)^{2} h^{6} \partial_{x} h+\mathcal{M} \frac{h^{2}}{(h+K)^{2}} \partial_{x} h\right. \\
& \left.+V_{r} \frac{h^{3}}{(h+K)^{3}} \partial_{x} h-\frac{1}{3} \operatorname{Re} \cos \beta h^{3} \partial_{x} h+\bar{S} h^{3} \partial_{x x x} h\right]+\epsilon \partial_{x}\left[\frac{5 \bar{E}}{24} \operatorname{Re} \sin \beta \frac{h^{4}}{h+K}\right] \\
& +\epsilon \bar{E} \operatorname{Pr} \frac{h^{3}}{(h+K)^{3}}\left[\frac{\bar{E}}{3(h+K)}+\frac{1}{120} \operatorname{Re} \sin \beta(7 h-15 K) h \partial_{x} h\right] \\
& +\epsilon \tau\left[\frac{1}{12} \bar{E} \operatorname{Pr} \frac{h^{4}}{(h+K)^{3}} \partial_{x} h+\frac{2}{15} \operatorname{Re} \sin \beta \partial_{x}\left(h^{5} \partial_{x} h\right)\right]=0,
\end{aligned}
$$

which describes the evolution of the liquid interface under the influence of gravity, hydrostatic pressure, thermocapillarity, evaporation, and gas shear as modeled by $\tau$. Note that this is the same equation obtained by Joo et al. [21] except for the additional terms proportional to $\tau$. We define $V_{r}=\bar{E}^{2} / D$, which is a parameter representing the strength of the vapor recoil forces, and $\mathcal{M}=K M / \operatorname{Pr}$, which represents the strength of the Marangoni forces as modified by evaporation effects. By making various assumptions within Eq. (7), several previously derived models can be recovered. When $\tau=0$, the exact equation derived by Joo et al. [21] is acquired. If then we set $\mathrm{Re}=0$, a generalization of the equation of Burelbach et al. [20] with first-order evaporation and convection terms is obtained. If we retain $\operatorname{Re}$ and set $\bar{E}=V_{r}=0$, the evaporative mass flux naturally goes to zero, $K$ takes the role of the Biot number, and Eq. (7) then describes a nonvolatile film evolving under the influence of gravity, thermocapillary forces, and gas shear. If further $\beta=\tau=0$, the equation derived by Davis [67] is retrieved, albeit without accounting for van der Waals forces. Finally, if $\tau=0$, the film is isothermal $\left(\bar{E}=V_{r}=\mathcal{M}=0\right.$ ), but $\beta \neq 0$, and Eq. (7) resembles Benney's [11] original equation for falling liquid films. 


\section{LINEAR STABILITY}

\section{A. Dispersion relationship derivation}

We now follow Joo et al. [21] and derive the expressions for a base flow that is spatially uniform but temporally evolving. We set $\partial_{x}=0$ in Eq. (7) to get

$$
\partial_{t} h_{0}+\frac{\bar{E}}{h_{0}+K}+\epsilon \bar{E}^{2} \operatorname{Pr} \frac{h_{0}^{3}}{3\left(h_{0}+K\right)^{4}}=0 .
$$

Solving Eq. (8), we obtain

$$
h_{0}(t)=\Omega-K+\epsilon \frac{\bar{E} \operatorname{Pr}}{3 \Omega}\left(\frac{K\left(2 K^{2}+6 K+3\right)}{1+K}+\frac{3 K^{2}}{2} \ln \frac{\Omega}{1+K}+\frac{K^{3}}{\Omega}-3 K \Omega-\bar{E} t\right)+O\left(\epsilon^{2}\right),
$$

where $\Omega=\left(K^{2}+2 K+1-2 \bar{E} t\right)^{1 / 2}$. Moreover, emulating Burelbach et al. [20], we employ the frozen interface approximation [20,68], which assumes that the timescale associated with the variation in base flow height is much larger than that associated with the perturbation's growth. In other words, we examine the stability of a base flow that is frozen in time. The amount of time it takes the liquid film governed by a particular parameter set to completely evaporate is called the dry-out time, denoted by $t_{D}$. An analytical expression for $t_{D}$ can be found by setting $h_{0}(t)=0$ and solving for $t$ in Eq. (9) to get

$$
t_{D}=\frac{1+2 K}{2 \bar{E}}
$$

which is identical to the expressions obtained in Refs. [20,21]. We define $t_{f}=t / t_{D}$ as the fraction of time until complete film evaporation, which in the subsequent stability analysis determines how much remains of the evaporating liquid film. We now assume that the interface height function $h(t)$ is perturbed by an infinitesimal harmonic perturbation such that

$$
h(t)=h_{0}(t)+\delta H e^{i(k x-\omega t)},
$$

where $H$ is the initial disturbance amplitude and $\delta$ is a small linearization parameter $(\delta \ll 1)$. Note that, due to the applied scaling [Eqs. (6)], here $k=k^{*} / \epsilon$ and $\omega=\omega^{*} / \epsilon$, where $k^{*}$ and $\omega^{*}$ are the wave number and angular frequency in the nonscaled dimensionless spatial and temporal coordinates $\left(x^{*}, t^{*}\right)$. Subtracting Eq. (8) from Eq. (7) and substituting Eq. (11), we obtain

$$
\begin{aligned}
\omega(k)= & -i \epsilon \bar{S} h_{0}^{3} k^{4}+i \epsilon\left[\frac{2}{15}(\operatorname{Re} \sin \beta)^{2} h_{0}^{6}+V_{r} \frac{h_{0}^{3}}{\left(h_{0}+K\right)^{3}}+\mathcal{M} \frac{h_{0}^{2}}{\left(h_{0}+K\right)^{2}}\right. \\
& \left.-\frac{1}{3} \operatorname{Re} \cos \beta h_{0}^{3}+\frac{2}{15} \operatorname{Re} \sin \beta h_{0}^{5} \tau\right] k^{2}+\left[\operatorname{Re} \sin \beta h_{0}^{2}+h_{0} \tau+\epsilon \bar{E}\left(\frac{5}{6} \operatorname{Re} \sin \beta \frac{h_{0}^{3}}{h_{0}+K}\right.\right. \\
& +\frac{7}{120} \operatorname{Re} \sin \beta \operatorname{Pr} \frac{h_{0}^{5}}{\left(h_{0}+K\right)^{3}}-\frac{5}{24} \operatorname{Re} \sin \beta \frac{h_{0}^{4}}{\left(h_{0}+K\right)^{2}}-\frac{1}{8} \operatorname{Re} \sin \beta \operatorname{Pr} \frac{h_{0}^{4}}{\left(h_{0}+K\right)^{3}} K \\
& \left.\left.+\frac{1}{12} \operatorname{Pr} \frac{h_{0}^{4}}{\left(h_{0}+K\right)^{3}} \tau\right)\right] k+i \frac{\bar{E}}{\left(h_{0}+K\right)^{2}}-i \epsilon \operatorname{Pr} \bar{E}^{2} \frac{h_{0}^{2}\left(3 K-h_{0}\right)}{3\left(h_{0}+K\right)^{5}} .
\end{aligned}
$$

Equation (12) is a dispersion relationship of the form $\omega(k)$ governing the linear dynamics of the liquid film. Note that the last two terms in Eq. (12) are not proportional to $k$, but rather are an algebraic addition to the imaginary part of the angular frequency $\omega$, as a function of the initial base state height $h_{0}$ [20,21]. This quantity is larger than zero for the range of parameters considered and therefore causes an increase in the perturbation's growth rate related not to the flow's stability, but instead to the reduction in base state height due to mass loss. Hence these two terms will be omitted from the dispersion relationship throughout the rest of this work whenever Eq. (12) is invoked. Note 
that in the following analysis the parameter values $\epsilon=0.15, \bar{S}=0.15, K=0.1$, and $\operatorname{Pr}=1$ are fixed unless otherwise stated.

\section{B. Temporal stability analysis}

For the purposes of temporal stability analysis it is assumed that the wave number $k$ is real and the angular frequency $\omega$ is complex. Under these assumptions, Eq. (12) can be written as

$$
\omega(k)=\omega_{r}+i \omega_{i},
$$

where $\omega_{r}=k c$ is the real angular frequency

$$
\begin{aligned}
\omega_{r}= & k\left[\operatorname{Re} \sin \beta h_{0}^{2}+h_{0} \tau+\epsilon \bar{E}\left(\frac{5}{6} \operatorname{Re} \sin \beta \frac{h_{0}^{3}}{h_{0}+K}+\frac{7}{120} \operatorname{Re} \sin \beta \operatorname{Pr} \frac{h_{0}^{5}}{\left(h_{0}+K\right)^{3}}\right.\right. \\
& \left.\left.-\frac{5}{24} \operatorname{Re} \sin \beta \frac{h_{0}^{4}}{\left(h_{0}+K\right)^{2}}-\frac{1}{8} \operatorname{Re} \sin \beta \operatorname{Pr} K \frac{h_{0}^{4}}{\left(h_{0}+K\right)^{3}}+\frac{1}{12} \operatorname{Pr} \frac{h_{0}^{4}}{\left(h_{0}+K\right)^{3}} \tau\right)\right]
\end{aligned}
$$

and $\omega_{i}$ is the temporal growth rate

$$
\omega_{i}=\epsilon\left[\frac{2}{15} \operatorname{Re} \sin \beta h_{0}^{6}\left(\operatorname{Re} \sin \beta+\frac{\tau}{h_{0}}\right)+\mathcal{M} \frac{h_{0}^{2}}{\left(h_{0}+K\right)^{2}}+V_{r} \frac{h_{0}^{3}}{\left(h_{0}+K\right)^{3}}-\frac{1}{3} \operatorname{Re} \cos \beta h_{0}^{3}-\bar{S} h_{0}^{3} k^{2}\right] k^{2} .
$$

Equations (14) show that the effect of $\tau$ depends on whether the liquid film is moving or stationary. Generally, for a moving film $(\beta \neq 0)$ positive values of $\tau$ increase the temporal growth rates corresponding to the band of unstable wave numbers. By contrast, the opposite is true for negative values of $\tau$. Furthermore, $\tau$ 's influence on the $H$ mode is much more pronounced than it is on the thermal instability mode. This is due to $\tau$ 's role as a multiplier of the flow's existing inertia as it appears as an algebraic variation to the film's velocity. For a stationary film $(\beta=0), \tau$ does not change the temporal growth rate since there is no base flow. As for the perturbation phase speed, $\tau$ will always change its value regardless of whether the film is flowing or not, whereby this change is contingent on the sign of $\tau$.

Figure 2(a) shows a plot of the maximum temporal growth rate across the range of unstable wave numbers versus the flow's Reynolds number. The figure shows the existence of sets of Reynolds number values for which the corresponding maximum temporal growth rates are positive. Moving along the Re axis, the first unstable set of Reynolds numbers corresponds to the thermal instability mode resulting from a combination of the Marangoni $S$ mode and the evaporative $E$ mode, whereas the second unstable set of values corresponds to the inertial $H$-mode instability, albeit modified by Marangoni and evaporation effects. The interconnection between the shear stress's role and the flow's inertia is highlighted by the different colored lines representing different values of $\tau$. At low Reynolds numbers, where the flow's inertia is weak, the effect of the shear stress is significantly weaker than its effect at higher Reynolds number where the flow is dominated by inertia.

The effect of the film thinning can be observed by comparing Fig. 2(a) to Fig. 2(b), which also shows a plot of the maximum temporal growth rate versus Reynolds number, however at reduced base flow thickness $\left(t_{f}=0.35\right)$. We find that, in general, the evaporation of the film and the consequent reduction in its base flow thickness lead to the strengthening of the thermal instability mode, as indicated by the expansion of the set of unstable values of Re and the increase in their corresponding maximum temporal growth rates. This occurs despite the thinner film being dominated by viscosity since the strength of the Marangoni and vapor recoil effects are significantly increased. These effects are proportional to the temperature gradient across the film, which becomes sharper as the film becomes thinner. In contrast, the thinning of the film results in the dampening of the $H$-mode instability due to the increased prominence of viscous forces. This appears in the plot as the contraction of the second set of unstable Reynolds numbers along with a decrease in their corresponding maximum temporal growth rates. 

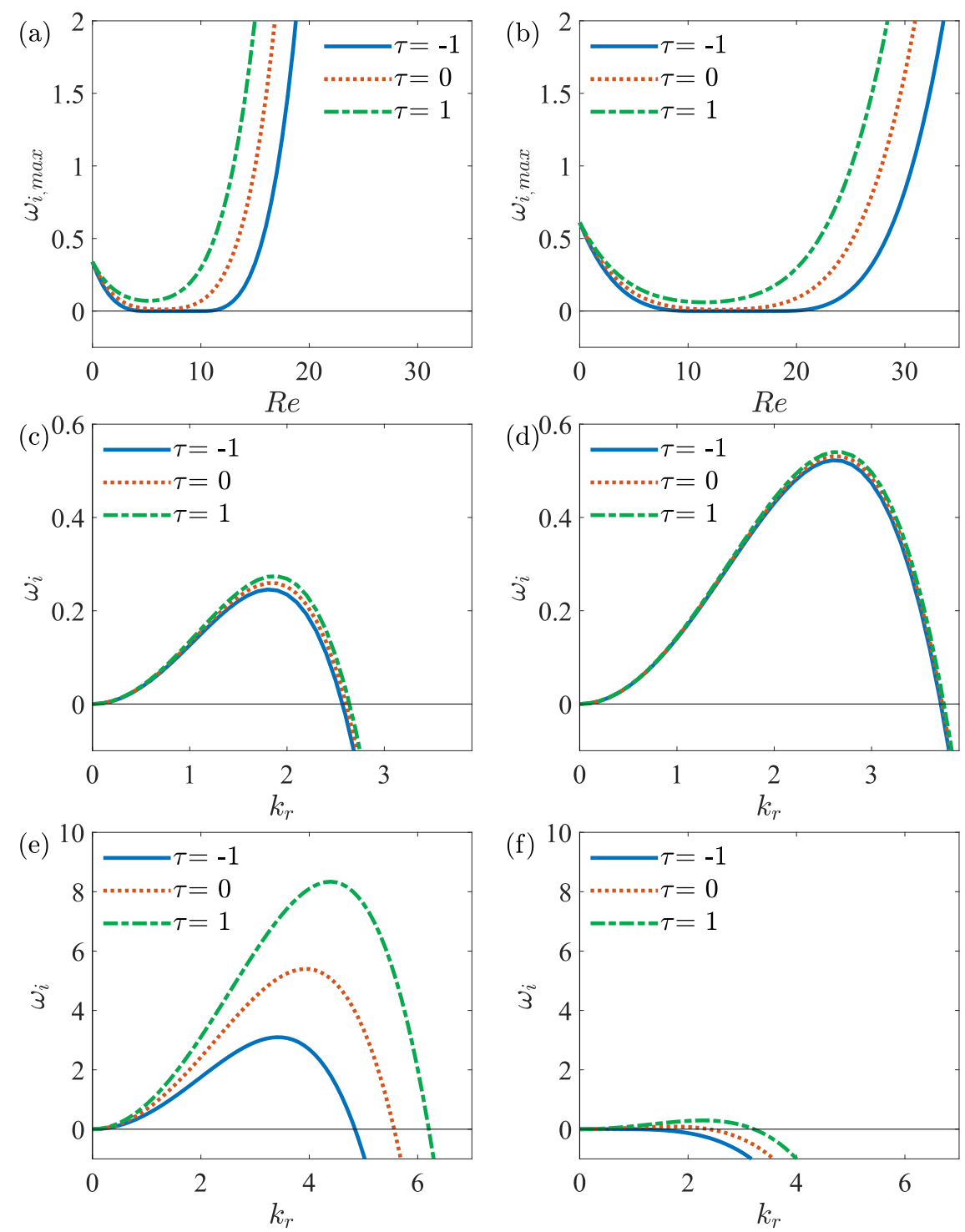

FIG. 2. Temporal growth rates for $\beta=25, \mathcal{M}=0.5, \bar{E}=0.1, V_{r}=1$. (a) $\omega_{i, \max }$ versus $\operatorname{Re}$ at $t_{f}=0$, (b) $\omega_{i, \max }$ versus Re at $t_{f}=0.35$, (c) $\omega_{i}$ versus $k$ for $\mathrm{Re}=0.5$ and $t_{f}=0$, (d) $\omega_{i}$ versus $k$ for $\operatorname{Re}=0.5$ and $t_{f}=0.35$, (e) $\omega_{i}$ versus $k$ for $\operatorname{Re}=20$ and $t_{f}=0$, and (f) $\omega_{i}$ versus $k$ for $\operatorname{Re}=0.5$ and $t_{f}=0.35$. Note that the parameters in (c) and (d) are in the range of the thermal instability mode, while those in (e) and (f) are in the range of the $H$-mode instability.

Figure 2(c) depicts a plot of the temporal growth rate versus wave number for different values of $\tau$ at a value of Reynolds number belonging to the thermal instability mode and at full film height. It can be seen that the shear stress's effect on the thermal instability's growth rate is rather small since its effect is proportional to the film's Reynolds number. On the other hand, Fig. 2(d) shows the temporal growth rates for the same film, but at a smaller thickness $\left(t_{f}=0.35\right)$, where the aforementioned strengthening of the thermal instability mode and the reduced impact of gas shear are clearly apparent.

The effects of the shear stress and film thinning on the $H$-mode instability are presented in Figs. 2(e) and 2(f). Figure 2(e) plots the temporal growth rate versus wave number for different 

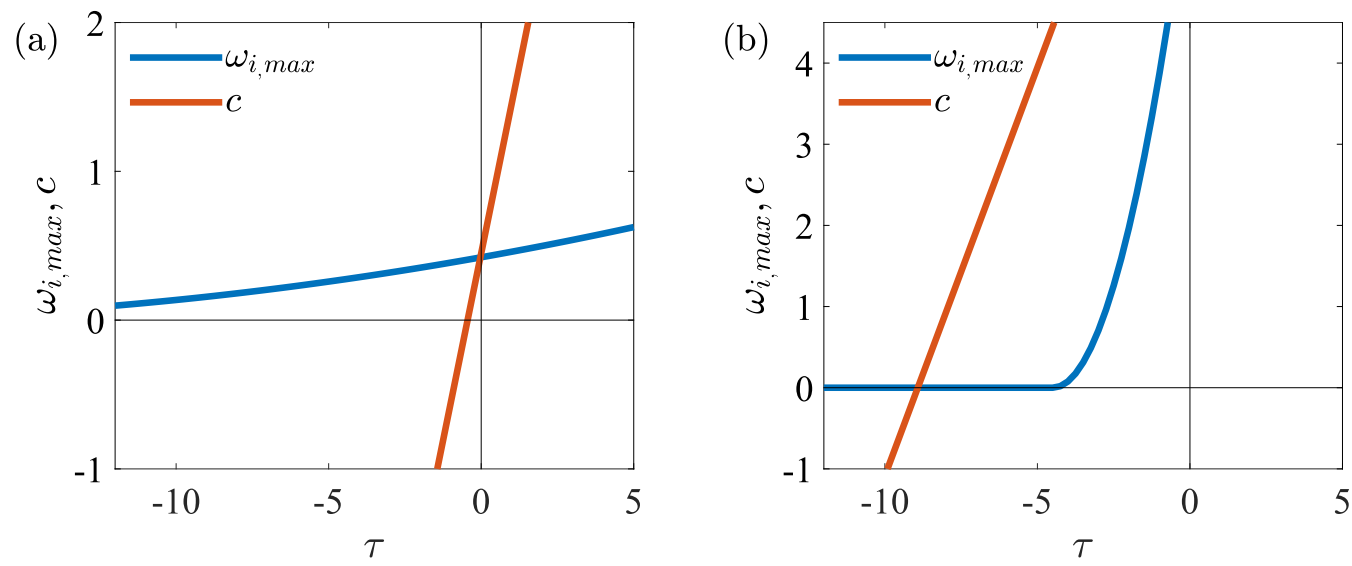

FIG. 3. Maximum temporal growth rate and perturbation phase speed versus $\tau$ for $\beta=25, \mathcal{M}=1, \bar{E}=$ $0.1, V_{r}=1, t_{f}=0$, and (a) $\operatorname{Re}=1$ (thermal mode instability) and (b) $\operatorname{Re}=20$ ( $H$-mode instability).

values of $\tau$ at $\operatorname{Re}=20$. The plots evidently show the significant impact of the shear stress on the $H$-mode instability, as seen in the large shifts in the temporal growth rate curves corresponding to the different values of $\tau$. Again, these effects are investigated at lower film thickness where Fig. 2(f) shows the strong suppressive nature of the negative values of $\tau$ on the $H$-mode instability. This suppressive behavior can be qualitatively linked to that in the work of Lavalle et al. [38] such that a negative value of $\tau$ is equivalent to a shearing gas counter-flowing alongside the liquid interface in a highly confined setting.

The contrast between the shear stress's effect on the thermal instability and that on the $H$-mode instability is highlighted in Fig. 3, where the maximum temporal growth rate and phase speed are depicted versus $\tau$ for $\mathrm{Re}=0.5$ [Fig. 3(a)], which belongs to the range of the thermal instability mode, and $\mathrm{Re}=20$ [Fig. 3(b)], which is in the range of the $H$-mode instability. In Fig. 3(a) it can be seen that for a range of negative values of $\tau$ the perturbation has a maximum temporal growth rate that is positive along with a negative phase speed, signifying an upstream-traveling perturbation. Contrarily, Fig. 3(b) shows that the maximum temporal growth rate is zero for perturbations with negative phase speed, so no upstream convection of $H$-mode instabilities occurs.

The reduction in film thickness due to evaporation results in it becoming increasingly dominated by viscosity, which resists not only the growth of the perturbations, but also their convection in the film. The thinner film's increased resistance to the convection of the perturbations is demonstrated in Fig. 4, which plots perturbation phase speed versus inclination angle [Fig. 4(a)] and $\tau$ [Fig. 4(b)] for an increasingly thinner film. In Figs. 4(a) and 4(b) we see a significant reduction in the slope of the plots, indicating a weakening of the convective nature of the flow with larger values of $t_{f}$. This demonstrates that the thinning of the film results in a significant reduction in the efficacy of flow drivers such as inclination angle and gas shear.

\section{Spatiotemporal stability analysis}

We investigate the spatiotemporal evolution of the film following a procedure based on the seminal work of Huerre and Monkewitz [45], where the spatiotemporal nature of the flow is determined by examining its long-time response to an infinitesimal impulse source. The distinction between the two types of instability is made by inspecting the temporal growth rate (denoted by $\sigma$ ) corresponding to the zero-group-velocity solution $(d \omega / d k \equiv V=0)$, known as the absolute growth rate and denoted by $\omega_{i, 0}$, where $\omega_{i, 0}<0$ indicates that the instability is convective and $\omega_{i, 0}>0$ indicates that it is absolute. Examples of impulse response analyses can be found in the literature for different flow systems (see, e.g., [69,70]). 

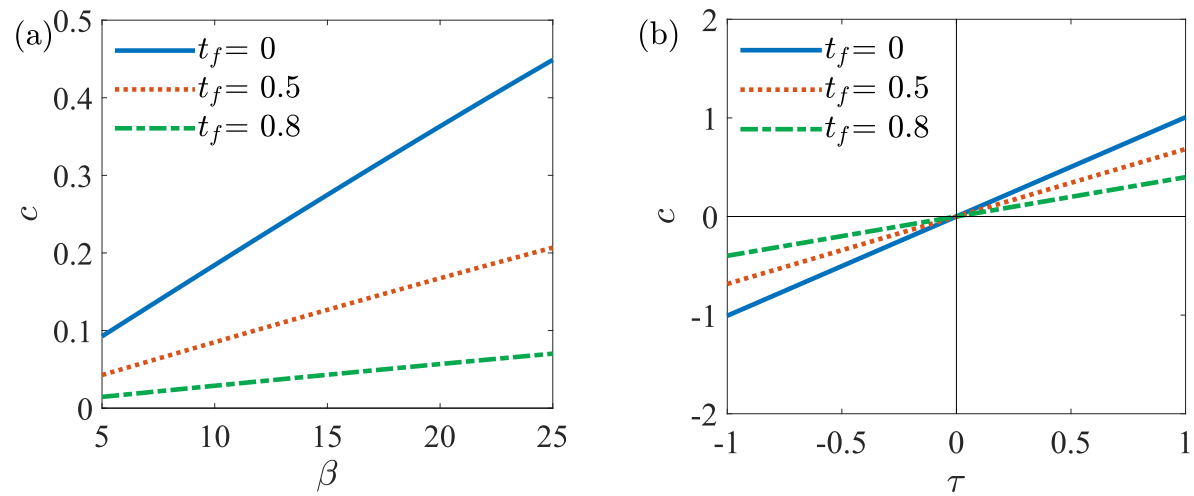

FIG. 4. Perturbation phase speed versus (a) inclination angle for $\tau=0$ and (b) $\tau$ for $\beta=0, \operatorname{Re}=1, \mathcal{M}=$ $0.5, \bar{E}=0.1, V_{r}=1$, and increasing values of $t_{f}$.

Equation (12) is used to obtain the full linear impulse response wave packets for different parameter sets, as shown in Fig. 5 where the effect of film evaporation on both the thermal and $H$-mode instabilities is investigated. Figure 5(a) depicts the impulse response wave packets for a film at full thickness and $\operatorname{Re}=1$, i.e., where the film is subject to the thermal instability mode. The

(a)

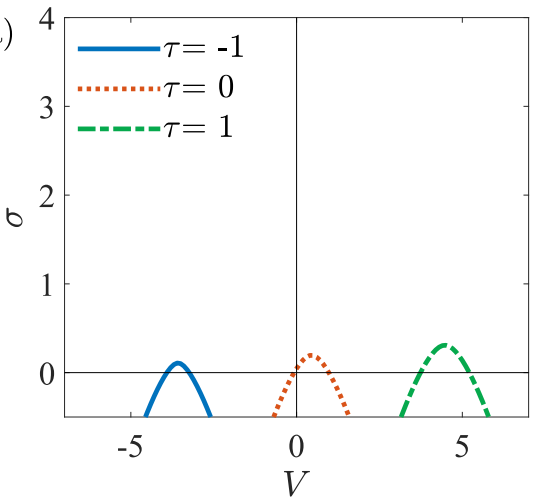

(c) 10

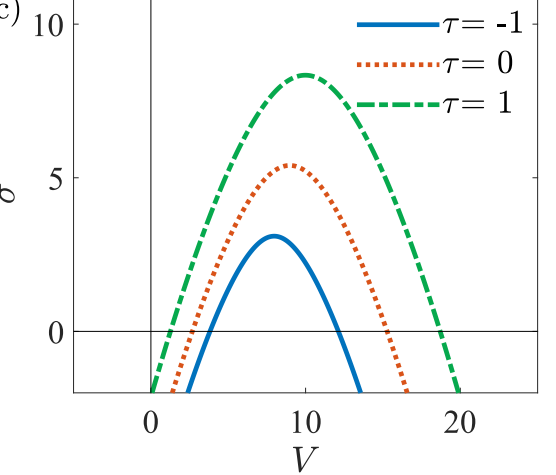

(b)

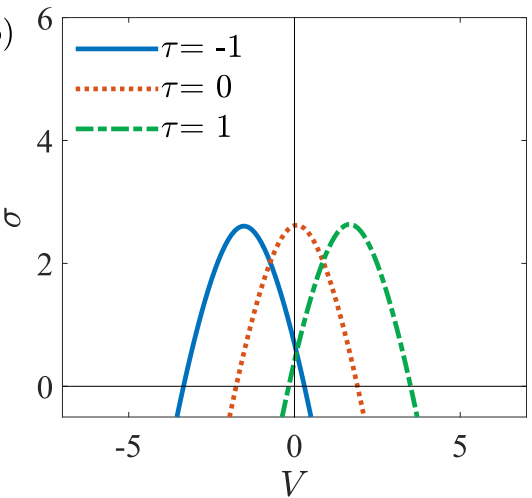

(d)

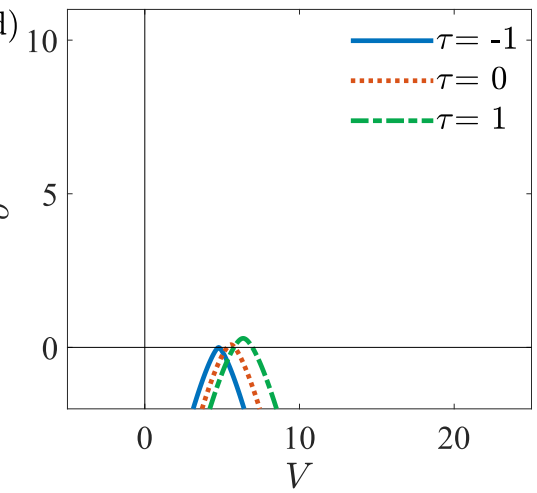

FIG. 5. Impulse response wave packets for $\beta=25, \mathcal{M}=0.5, \bar{E}=0.1, V_{r}=1$, and (a) $\operatorname{Re}=1$ and $t_{f}=0$, (b) $\operatorname{Re}=1$ and $t_{f}=0.8$, (c) $\operatorname{Re}=20$ and $t_{f}=0$, and (d) $\operatorname{Re}=20$ and $t_{f}=0.35$. Note that the parameters in (a) and (b) are in the range of the thermal instability mode, while those in (c) and (d) are in the range of the $H$-mode instability. 
plot shows three distinct wave packets corresponding to three values of $\tau$, where the wave packet associated with $\tau=1$ has the highest peak due to the shear stress's contribution to the growth rate. The central wave packet corresponding to $\tau=0$ has its edges on opposite sides of the $V=0$ axis, resulting in $\omega_{i, 0}>0$, and thus represents a perturbation which is absolute in nature. On the other hand, the two outermost wave packets corresponding to $\tau=-1$ and $\tau=1$ have both fronts on the same side of the $V=0$ axis, resulting in $\omega_{i, 0}<0$ and indicating that they represent perturbations which are convected by the flow upstream and downstream, respectively.

The effect of the film thinning on the impulse response is shown in Fig. 5(b), where $t_{f}=0.8$. The aforementioned amplification of the thermal instability mode is reproduced by our spatiotemporal analysis as all the wave packets are noticeably more prominent. Furthermore, at this reduced film thickness all three wave packets include positive values of $\omega_{i, 0}$ and therefore correspond to absolute instabilities. This indicates that the thinning of the film caused a convective-absolute transition of the thermal instability mode in the cases of $\tau= \pm 1$. Additionally, noting that the group velocity at the center of the impulse response wave packets in Fig. 5 corresponds to the perturbation phase speed $c$ from the temporal stability analysis [71], we notice again here that the thinning of the film decreases the phase speed of the perturbations, as observed in Fig. 4. However, comparing the wave packets in Figs. 5(a) and 5(b), we observe that the edges of the wave packets corresponding to $\tau=0$ and $\tau=1$ have significantly larger velocities, which implies that the overall progression of the thermal instability mode is enhanced by the reduction in film thickness. In other words, the thinning of the film hinders the convection of the thermal instability mode by the shearing gas or the flow's inertia, but actually increases the rate at which it spreads via its inherent instability mechanism.

Figure 5(c) presents the impulse response wave packets for a film at full thickness and $\operatorname{Re}=20$, i.e., where the film is subject to the $H$-mode instability. The strong effect that the shear stress has on the temporal growth rate of the $H$-mode instability is again clearly demonstrated by the significant change in the curves as the value of $\tau$ is varied. The considerable suppressive effect of the film thinning on the $H$-mode instability becomes apparent by comparing Figs. 5(c) and 5(d), which differ only by the value of $t_{f}: t_{f}=0$ [Fig. 5(c)] and $t_{f}=0.35$ [Fig. 5(d)].

The interaction between the shear stress and the effects of the film's evaporation can also be visualized by plotting the absolute growth rate in the (Re, $V_{r}$ ) space as shown in Fig. 6 where the blue, red, and green regions correspond to convectively unstable flow, absolutely unstable flow, and stable flow, respectively. Figures 6(a)-6(c) plot the absolute growth rate in a film being sheared by a counterflowing gas $(\tau=-1)$ for different values of $t_{f}$. Note the existence of two regions corresponding to convectively unstable flow: (i) The region on the left contains $\left(\operatorname{Re}, V_{r}\right)$ values belonging the thermal instability mode and (ii) the region on the right contains $\left(\operatorname{Re}, V_{r}\right)$ values belonging to the $H$-mode instability. In the former (latter) region the perturbation is convected upstream (downstream) by the shear stress (flow). In between there is a parameter set resulting in absolutely unstable flow where the downstream convection by the flow's inertia is balanced by the negative $\tau$ value representing a counterflowing shearing gas. As the value of $t_{f}$ is increased, the parameter set corresponding to the absolutely unstable flow expands at the expense of those associated with convective instability. Similarly, the parameter set corresponding to stable flow expands as well, raising the critical Re associated with the onset of the $H$-mode instability outside the parameter set we considered. In Fig. 6(c) where $t_{f}=0.7$, the amplified convective thermal instability mode persists up to Reynolds numbers of $O(10)$.

On the other hand, Figs. 6(d)-6(f) plot the absolute growth rate in a film being sheared by a coflowing gas $(\tau=1)$ for different values of $t_{f}$. Due to the positive value of $\tau$, the parameter space is initially dominated by convectively unstable flow as the parameter set resulting in absolutely unstable flow is limited to the upper left corner. As the film thins the parameter sets corresponding to stable and absolutely unstable flow grow into regions of the parameter space previously occupied by convectively unstable flows. 

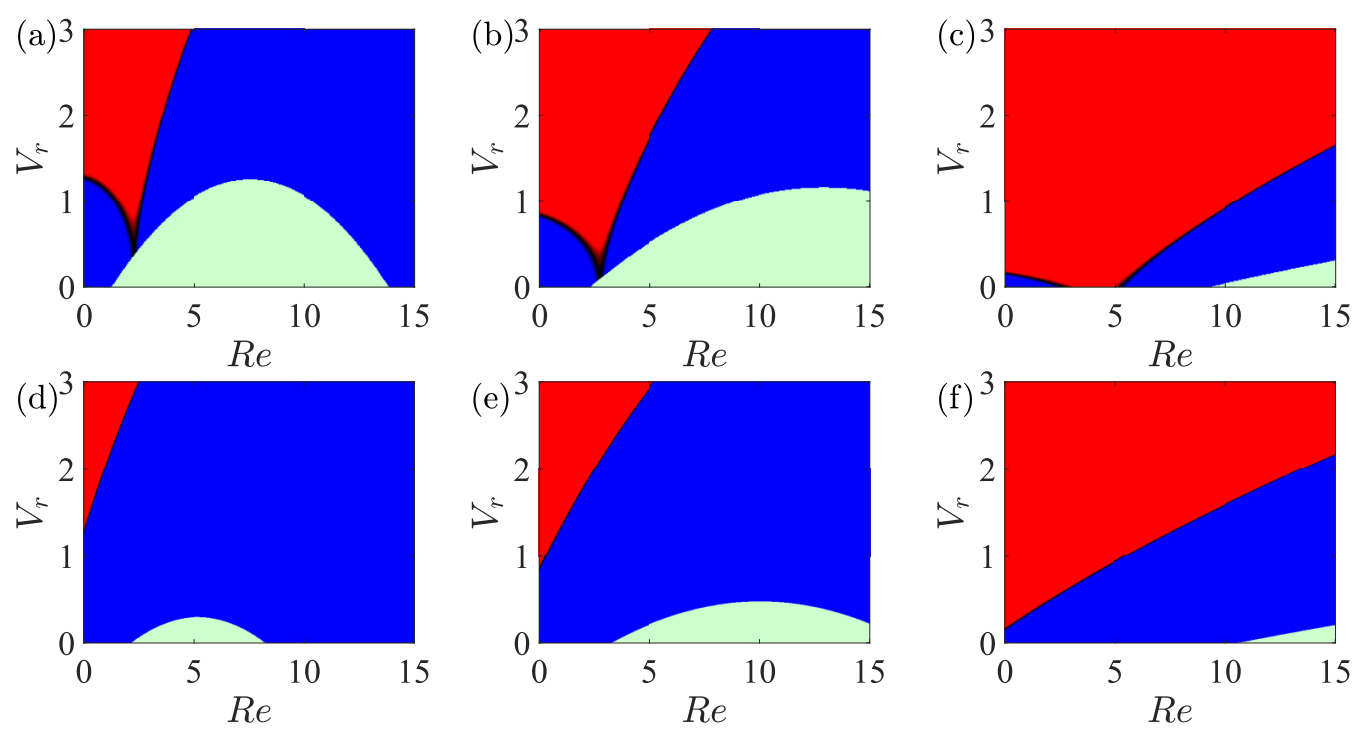

FIG. 6. Absolute growth rate in the $\left(\operatorname{Re}, V_{r}\right)$ space for $\beta=25, \mathcal{M}=0.5, \bar{E}=0.1$, and (a) $\tau=-1$ and $t_{f}=0$, (b) $\tau=-1$ and $t_{f}=0.3$, (c) $\tau=-1$ and $t_{f}=0.7$, (d) $\tau=1$ and $t_{f}=0$, (e) $\tau=1$ and $t_{f}=0.3$, and (f) $\tau=1$ and $t_{f}=0.7$. The red, blue, and green regions indicate absolutely unstable, convectively unstable, and stable flow, respectively.

\section{NONLINEAR EVOLUTION}

The linear stability analysis in Sec. III is only valid when the perturbation amplitude is small. To relax this constraint we solve Eq. (7) numerically as an initial value problem using MATLAB [72]. A perturbation in the form of a Gaussian wave packet localized about $x=0$ is introduced such that the initial interface is described by $h(x, 0)=h_{0}+A \exp \left(-10 x^{2}\right)$, where $h_{0}$ is the initial flat film height and $A$ is the Gaussian wave packet's maximum amplitude. For the numerical simulations we keep $\epsilon=0.15, \bar{S}=0.15, K=0.1$, and $\operatorname{Pr}=1$.

\section{A. Solver validation}

The solver is validated by comparing the temporal growth rates obtained analytically to those computed from numerical data resulting from solving Eq. (7) for an interface disturbed initially by a small-amplitude perturbation. For a particular mode within the Gaussian wave packet with an amplitude $\hat{h}(k, t)$, the numerical and analytical temporal growth rates are related by

$$
\frac{\dot{\hat{h}}}{\hat{h}}=\omega_{i} \text {. }
$$

To compute the left-hand side of Eq. (15) we find $\hat{h}(k, t)$ via the discrete Fourier transform of $h(x, t)$ as

$$
\hat{h}(k, t)=2|\hat{f}(k, t)| /(n-1),
$$

where

$$
\hat{f}(k, t)=\sum_{j=1}^{n-1} h(j, t) e^{-2 \pi i(j-1)(k-1) /(n-1)}
$$




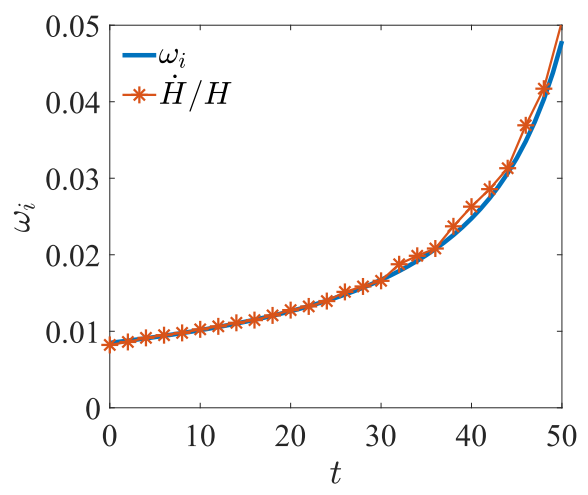

FIG. 7. Comparison of the analytical and numerical temporal growth rates for $\beta=5, \operatorname{Re}=1, \mathcal{M}=0.2$, $\bar{E}=0.01, V_{r}=0.2$, and $t_{f}=0$. The initial interface in the numerical simulation is described by $h(x, 0)=$ $h_{0}+A \exp \left(-10 x^{2}\right)$, where $A=0.005$.

and $n$ is the total number of discrete points in $h(x, t)$ and $k \geqslant 1$. We subsequently take its derivative numerically to obtain $\dot{\hat{h}}$. The right-hand side of Eq. (15) is computed from Eq. (14b).

An example of this validation is shown in Fig. 7, where the error between the numerical and analytical values was found to be approximately equal to $1 \%$ during linear perturbation growth. Further validation in the nonlinear regime was conducted by comparing the our simulation results to those in Ref. [21], where excellent agreement was obtained.

\section{B. Interface evolution}

Figure 8 shows the results of simulating an evaporating film flowing down an inclined plane while being assisted by a coflowing shearing gas, represented by a positive value of $\tau$, and with $A=0.1$. The parameter set was chosen specifically such that the evaporation of the film and the growth of the perturbation are sufficiently slow to allow observing several stability regimes. We observe that the initial perturbation amplitude decays as it travels downstream; however, as the film's thickness decreases the thermal instability mode arises and the perturbation begins to grow. At this stage the instability is convective in nature and is carried downstream by the flow. Further thinning of the film causes the perturbation to undergo a convective-absolute transition, and the instability can be seen to start spreading in the upstream direction as well. Notably, the reduction in the perturbation's

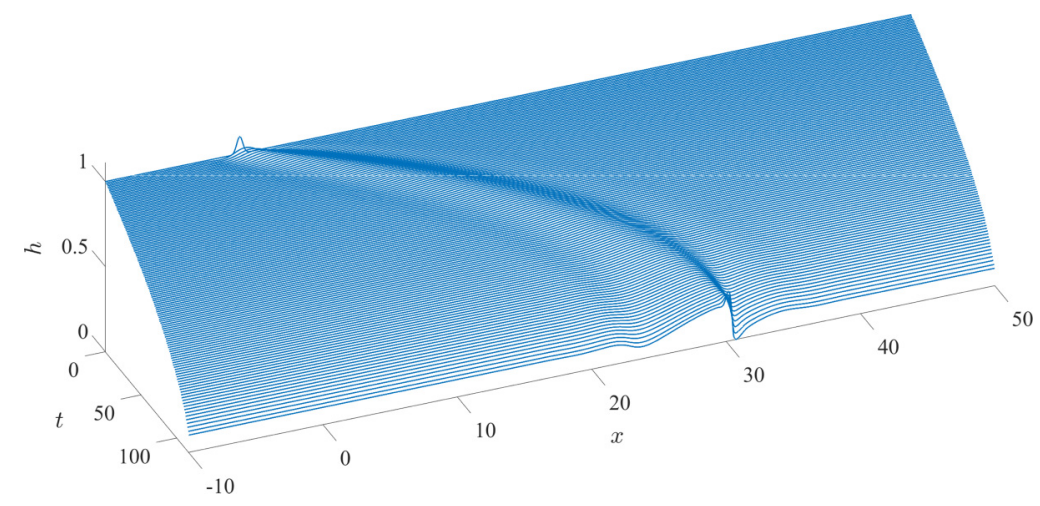

FIG. 8. Evolution of the fluid interface for $A=0.1, \beta=5, \operatorname{Re}=1, \mathcal{M}=0.1, \bar{E}=0.005, V_{r}=0.2, \tau=$ 0.3 , and $t_{f}=0$. 

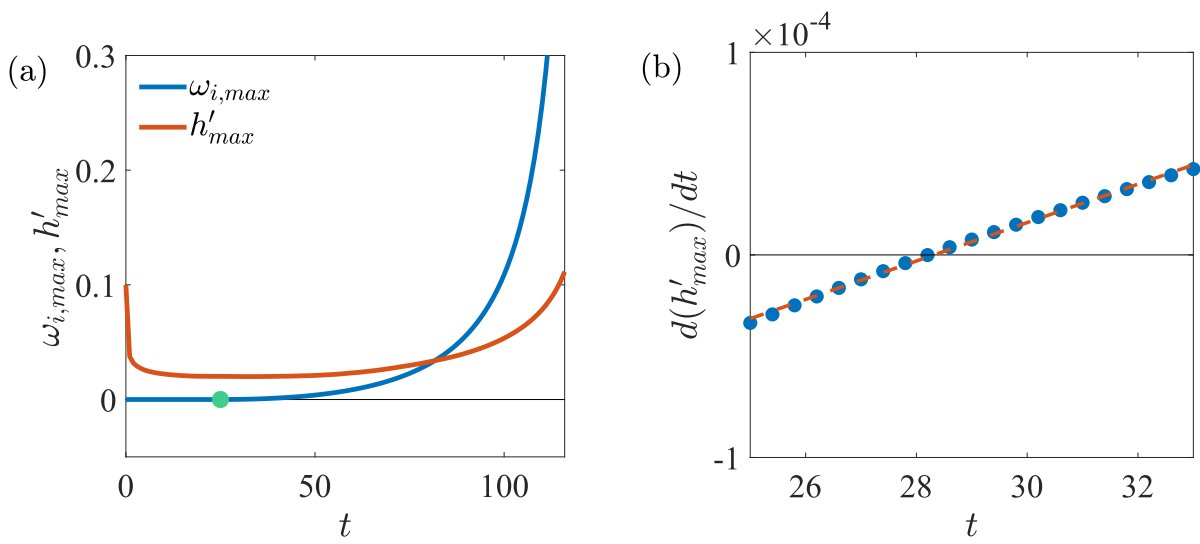

FIG. 9. Temporal stability analysis for $\beta=5, \operatorname{Re}=1, \mathcal{M}=0.1, \bar{E}=0.005, V_{r}=0.2, \tau=0.3$, and $t_{f}=$ 0. (a) $\omega_{i, \max }$ and $h_{\max }^{\prime}$ versus time and (b) $d h_{\max }^{\prime} / d t$ versus time.

phase speed is clearly demonstrated by the reduction in the rate of progression of the wave packet's center when observed in the $(x, t)$ plane.

\section{Temporal evolution}

The dynamics exhibited in Fig. 8 are investigated first by a temporal stability analysis. The temporal evolution of the perturbation is examined in the linear regime by computing the maximum temporal growth rate using Eq. (14b) and in the nonlinear regime by tracking the maximum perturbation height, denoted by $h_{\max }^{\prime}$, which represents the maximum deviation of the film's interface from the flat base flow such that $h_{\max }^{\prime}(t)=\left|h(x, t)-h_{0}(t)\right|$. Both these quantities are plotted versus time in Fig. 9(a), where $\omega_{i, \max }$ is found to be zero up to $t=24.5$ (marked by the green circle), indicating that the fluid film is indeed initially linearly stable. The thinning of the film beyond this point results in the flow becoming temporally unstable due to the onset of the thermal instability mode. Furthermore, the same qualitative behavior predicted by linear stability analysis is observed in the nonlinear regime, as $h_{\max }^{\prime}$ initially decreases and then starts to increase. We attempt to determine this inflection point, i.e., the point in time at which $h_{\max }^{\prime}$ begins to grow, by numerically computing $d\left(h_{\max }^{\prime}\right) / d t$ and fitting the data around the transition point linearly. We consider the intercept of the linear fitting function with the $t$ axis to be the transition point from stable to unstable flow, which occurs at $t=28.3,15.5 \%$ higher than the value predicted by linear stability theory. This difference is attributed to nonlinear effects.

\section{Spatiotemporal evolution}

The wave packet's spatiotemporal evolution within the flow domain can be observed by tracking the perturbation's edges, which are defined by the two points on the interface where $\left|\partial_{x} h\right|$ increases beyond a small tolerance, indicating the existence of a perturbed interface deviating from the flat base flow. The loci of these points are plotted against time in Fig. 10(a), which shows that the wave packet's edges both initially travel downstream, forming a convective instability. Subsequently, the rear edge begins to slow down relative to the front edge, eventually reversing direction and traveling upstream, indicating the transition to absolutely unstable flow. The colored circles mark the wave packet's edges at four points in time corresponding to the interface profiles in Fig. 10(b). Comparing the four interface profiles, the transition from convective to absolute instability is apparent.

The spatiotemporal dynamics in the nonlinear regime are further examined by generating the complete impulse response wave packets by numerically computing the temporal growth rate along each ray $(V=x / t)$. We consider the perturbation amplitude at any point along the streamwise 

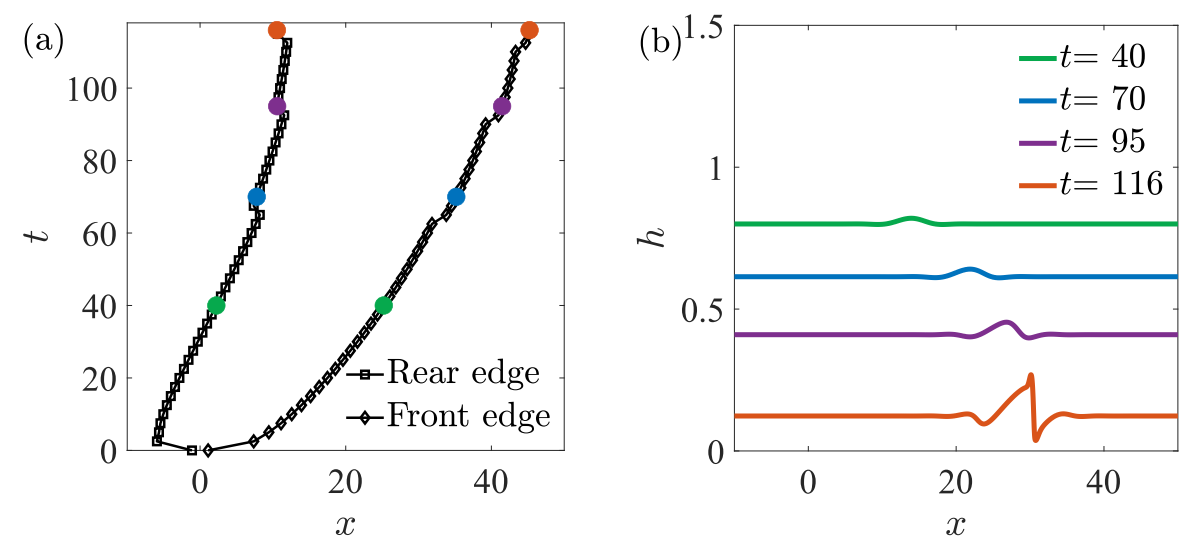

FIG. 10. Visualization of wave-packet spreading in the flow domain for $\beta=5, \operatorname{Re}=1, \mathcal{M}=0.1, \bar{E}=$ $0.005, V_{r}=0.2, \tau=0.3$, and $t_{f}=0$. (a) Front and rear wave-packet edge positions in time. (b) The evolution of the interface profile through the convective-absolute transition.

coordinate as the maximum perturbation height $h_{\max }^{\prime}(x, t)$, a quantity which is shown to behave asymptotically [73] as

$$
h_{\max }^{\prime} \propto t^{-1 / 2} e^{\sigma(V) t}, \quad V=x / t=\mathrm{const} \quad \text { for } t \rightarrow \infty,
$$

where again $\sigma$ is the temporal growth rate along a given ray $V=x / t=$ const, which can be found analytically as

$$
\sigma(V) \sim \frac{d}{d t}\left\{\ln \left[t^{1 / 2} h_{\max }^{\prime}(V t, t)\right]\right\} \quad \text { for } t \rightarrow \infty .
$$

This expression can be discretized in a form suitable for numerical computation as

$$
\sigma(V) \approx \frac{\ln \left[h_{\max }^{\prime}\left(V t_{2}, t_{2}\right) / h_{\max }^{\prime}\left(V t_{1}, t_{1}\right)\right]}{t_{2}-t_{1}}+\sigma_{0}\left(t_{2}, t_{1}\right),
$$

where $\sigma_{0}\left(t_{1}, t_{2}\right)=\ln \left(t_{2} / t_{1}\right) /\left[2\left(t_{2}-t_{1}\right)\right]$ is a finite-time correction term. For further details, see Ref. [74].

Note that the analytical absolute growth rate is obtained under the notion that the base flow height does not vary during the growth of the perturbation, i.e., under the frozen interface assumption $[20,68]$. In order to allow comparison with linear theory, we enforce this assumption when numerically computing the nonlinear absolute growth rate by simulating the interface evolution at a constant base flow height $(\bar{E}=0)$. Furthermore, the temporal growth rate was monitored over time to ensure that the perturbations were growing nonlinearly at the time when the nonlinear impulse response was acquired. Additionally, the resulting impulse responses were obtained at a value of $t_{2}$ large enough to ensure the saturation of the computed values of $\sigma$ [see Eq. (18)]. The value of $\omega_{i, 0}$ was approximated by linearly interpolating between the two peaks closest to the $V=0$ axis, which is sufficiently accurate for our qualitative analysis.

Figure 11(a) plots the linear and nonlinear absolute growth rates versus film thickness for the range of values of $h_{0}$ where the flow is temporally unstable. We observe that initially, when the film is relatively thick, the nonlinear absolute growth rate is higher than its linear counterpart, indicating that finite instabilities are less convective than infinitesimal ones for this set of parameters. However, as the film height decreases, the linear and nonlinear absolute growth rates converge. Note that the two growth rates appear to slightly diverge again towards the end of the range of $h_{0}$ values considered. However, this is due to the film rupturing at a time which is too early to allow accurate estimation of the growth rates. 

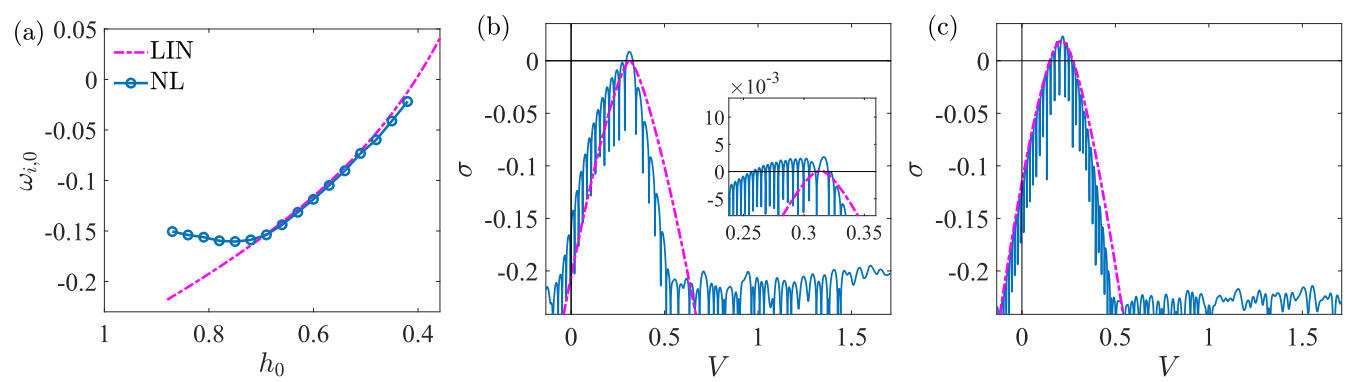

FIG. 11. Analytical (linear) and numerical (nonlinear) impulse responses for $\beta=5, \operatorname{Re}=1, V_{r}=0.2$, $\mathcal{M}=0.1, \bar{E}=0.005, \tau=0.3$, and $t_{f}=0$. (a) Absolute growth rate versus $h_{0}$. (b) Linear and nonlinear impulse response wave packets for $h_{0}=0.84$. (c) Linear and nonlinear impulse response wave packets for $h_{0}=0.6$. Note that LIN (magenta dashed lines) and NL (blue solid lines) indicate linear and nonlinear data, respectively.

The impulse response wave packet corresponding to $h_{0}=0.84$ is depicted in Fig. 11(b), where the large disparity between the linear and nonlinear responses behind the difference in absolute growth rate is seen. The inset of Fig. 11(b) details the wave packets' peaks and demonstrates the significant difference in the upstream and downstream front velocities of the linear and nonlinear responses. According to Dee and Langer's criterion [75], this implies that in the case of finite perturbations, both front velocities are nonlinearly selected as the nonlinear wave packet's saturation produced wave fronts with velocities higher than those which would result from an infinitesimal perturbation. On the other hand, the impulse response wave packet corresponding to $h_{0}=0.6$ is shown in Fig. 11(c), where we observe that the saturated nonlinear wave packet's fronts match their linear counterparts, indicating that the front velocities are linearly selected, which suggests that for this thinner film the front dynamics are dictated by infinitesimal modes governed by the linear dispersion relationship (12). The convergence of the nonlinear front velocities towards their linear equivalent as the film thins suggests that this effect is related to the strength of the instability, since the thinning of the film results in a significantly stronger thermal instability mode. This is investigated by generating both the linear and nonlinear impulse responses for a constant film thickness of $h_{0}=1$ and increasingly stronger instabilities (higher values of $\mathcal{M}$ and $V_{r}$ ) (see Fig. 12). Both the linear and nonlinear absolute growth rates are plotted versus increasing values of $\mathcal{M}$ in Fig. 12(a). The same trend observed in Fig. 11 appears here as the nonlinear value of $\omega_{i, 0}$ is initially larger its linear counterpart. However, again this deviation decreases with larger values of $\mathcal{M}$ until the eventual convergence of the linear and nonlinear absolute growth rates. This is confirmed by plotting the linear and nonlinear impulse response wave packets for $\mathcal{M}=0.32$, as shown in Fig. 12(b), where the disparity between the linear and nonlinear responses is apparent, and those corresponding to $\mathcal{M}=0.64$, as shown in Fig. 12(c), where their concurrence is demonstrated.

Moreover, Fig. 12(d) shows the linear and nonlinear absolute growth rates for a film with a constant thickness $h_{0}=1$ and increasing values of $V_{r}$. Once more we observe initially higher values of the nonlinear absolute growth rate and the subsequent convergence to the linear value as $V_{r}$ is increased. This is confirmed by plotting the linear and nonlinear impulse response wave packets in Figs. 12(e) and 12(f), corresponding to $V_{r}=0.42$ and $V_{r}=0.86$, respectively.

\section{Rupture analysis}

Thermal instabilities can lead to the rupture of the fluid film, which can have significant negative effects on applications sensitive to the film's integrity. Therefore, we examine the effects of the shear stress on rupture location (denoted by $x_{r}$ ) and rupture time (denoted by $t_{r}$ ). We expect a shearing gas to play a role in mitigating these issues by either preventing rupture altogether or altering the evolution of the interface such that film rupture is delayed until the disturbance exits the region in 

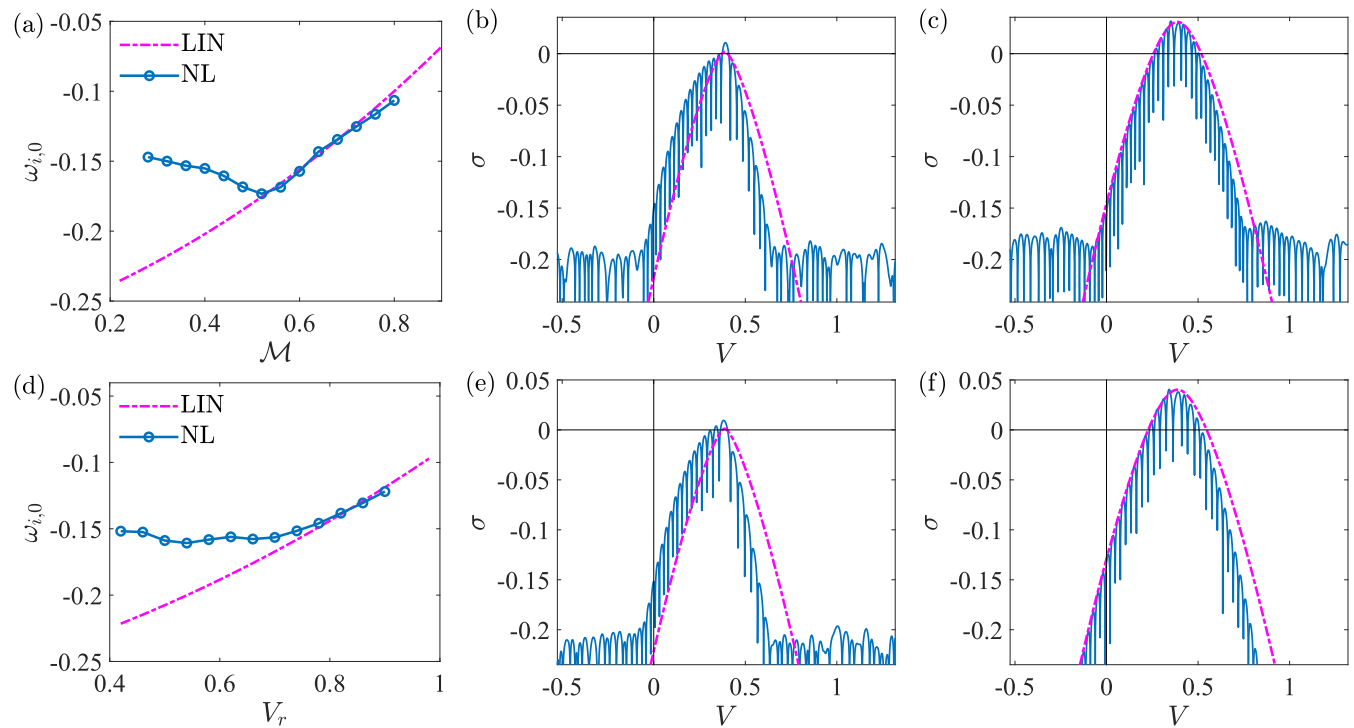

FIG. 12. Analytical (linear) and numerical (nonlinear) wave-packet impulse responses for $\beta=5, \operatorname{Re}=$ $1, \bar{E}=0.005, \tau=0.3$, and $t_{f}=0$. (a) Absolute growth rate versus $\mathcal{M}$ for $V_{r}=0.2$. Linear and nonlinear impulse response wave packets for (b) $\mathcal{M}=0.32$ and (c) $\mathcal{M}=0.64$. (d) Absolute growth rate versus $V_{r}$ for $\mathcal{M}=0.1$. Linear and nonlinear impulse response wave packets for (e) $V_{r}=0.42$ and (f) $V_{r}=0.86$. Note that LIN (magenta dashed lines) and NL (blue solid lines) indicate linear and nonlinear data, respectively.

which it would be detrimental to the system. Note that in the presence of more than one simultaneous effective rupture location, the one closest to the origin of the perturbation $(x=0)$ is considered to be the rupture point.

In Fig. 13(a) rupture location $x_{r}$ is plotted against increasing values of both $\mathcal{M}$ and $V_{r}$. We find that increasing either parameter causes the rupture location to shift closer towards the origin. This occurs since an increase in $\mathcal{M}$ or $V_{r}$ enhances the perturbation's temporal growth rate, allowing less time for it to travel before film rupture. However, this trend does not continue across the range of values of $\mathcal{M}$ and $V_{r}$ we considered, as at a certain value of either parameter the rupture location is abruptly shifted downstream before again resuming its upstream trend. On the other hand, rupture
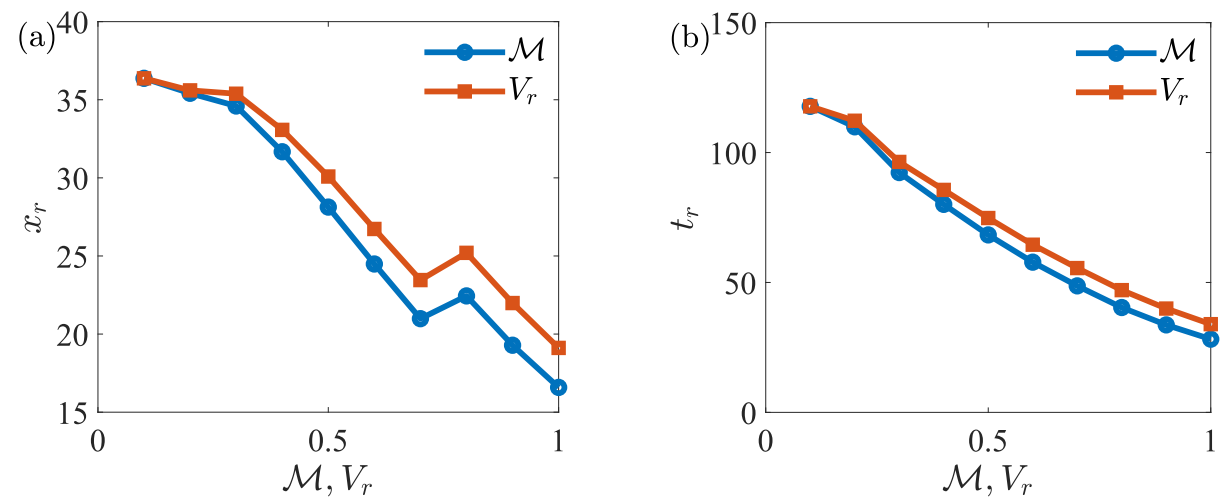

FIG. 13. Rupture (a) location and (b) time versus $\mathcal{M}$ and $V_{r}$ for $\beta=5, \operatorname{Re}=1, \bar{E}=0.005, \tau=0.5$, and $t_{f}=0$. 

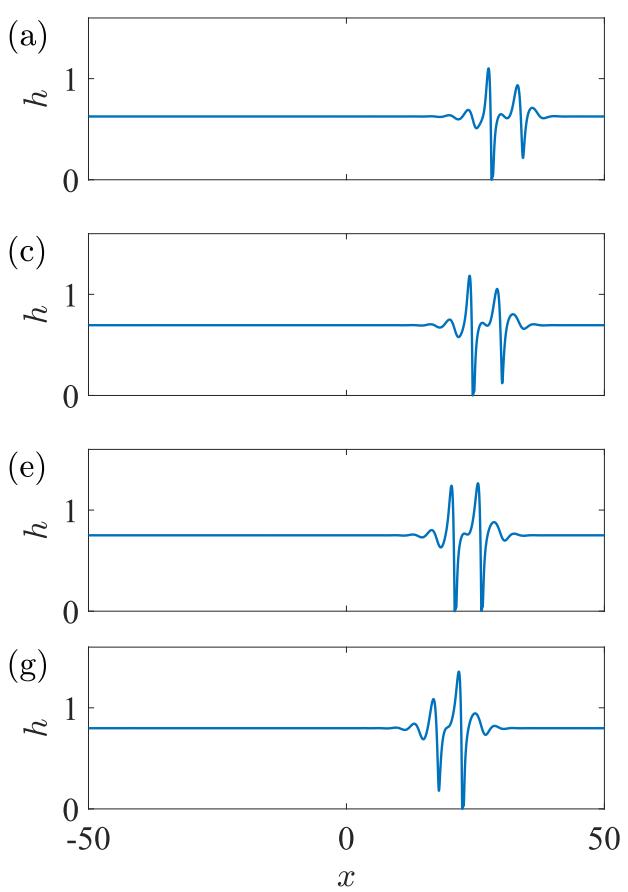
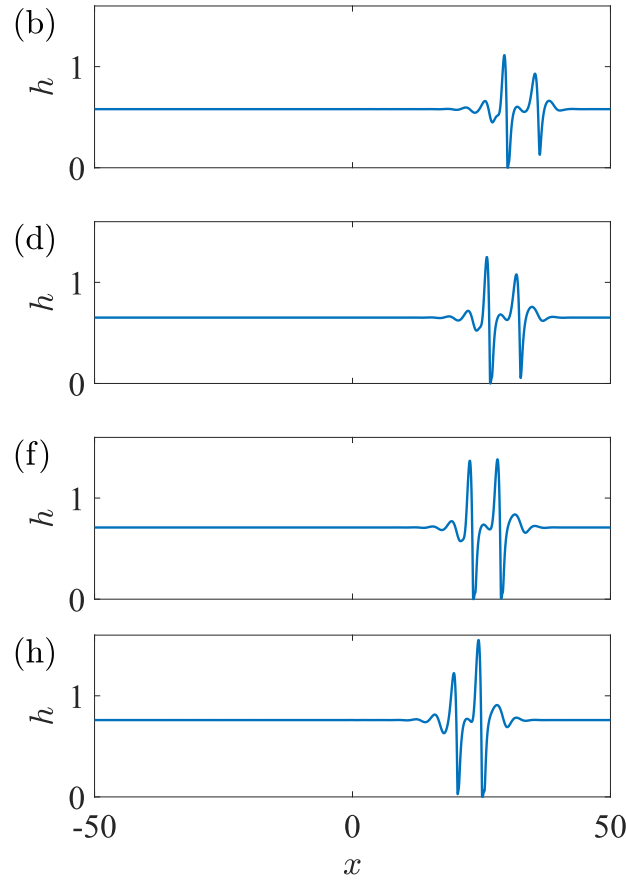

FIG. 14. Interface profiles at film rupture for $\beta=5, \operatorname{Re}=1, \bar{E}=0.005, \tau=0.5, t_{f}=0$, and (a) $V_{r}=0.1$ and $\mathcal{M}=0.6$, (c) $V_{r}=0.1$ and $\mathcal{M}=0.7$, (e) $V_{r}=0.1$ and $\mathcal{M}=0.8$, (g) $V_{r}=0.1$ and $\mathcal{M}=0.9$, and (b) $\mathcal{M}=0.1$ and $V_{r}=0.6$, (d) $\mathcal{M}=0.1$ and $V_{r}=0.7$, (f) $\mathcal{M}=0.1$ and $V_{r}=0.8$, and (h) $\mathcal{M}=0.1$ and $V_{r}=0.9$.

time does not display this harmonic behavior as $t_{r}$ decreases monotonically across the considered range of $\mathcal{M}$ and $V_{r}$ values [see Fig. 13(b)].

We investigate this further by plotting the interface profiles at rupture for different values of $\mathcal{M}$ and $V_{r}$, as shown in Fig. 14. In particular, in Figs. 14(a), 14(c), 14(e), and 14(g) we keep $V_{r}=0.1$ but vary the value of $\mathcal{M}$, while in Figs. 14(b), 14(d), 14(f), and 14(h) the value of $\mathcal{M}=0.1$ is retained while the value of $V_{r}$ is increased. The rupture profile corresponding to $\mathcal{M}=0.6$ is presented in Fig. 14(a) and shows that film rupture occurs towards the rear edge of the perturbation, while the front edge remains far from rupture. When the value of $\mathcal{M}$ is increased to 0.7 , the same dynamic is preserved; however, the front edge of the perturbation is significantly closer to rupture as it occurs, as seen in Fig. 14(c). Further increases in the value of $\mathcal{M}$ show that the rupture location is changed from the rear of the perturbation towards to the front as the value of $\mathcal{M}$ crosses a critical threshold [see Figs. 14(e) and 14(g)]. Additional increases in the value of $\mathcal{M}$ restore the original trend of rupture location moving closer towards the origin of the disturbance. Interestingly, the same dynamic is reproduced when the value of $\mathcal{M}$ is kept constant while the value of $V_{r}$ is increased. Here again we observe a shift in the evolution dynamics as the strength of the thermal instability mode is increased, akin to that observed in Sec. IV B 2.

\section{Self-similarity analysis}

Self-similar analysis of fourth-order thin-film-type equations has been performed extensively in the past (see Refs. $[53,55,56,76,77]$ ) and is a useful framework within which to understand and predict the rupture of thin films. The solutions of Eq. (7) rupture in finite or infinite time or exhibit other long-time behavior depending on the presence or absence of thermal disequilibrium $K$, evaporative mass loss $\bar{E}$, and shear $\tau$. In the absence of mass loss $(\bar{E}=0)$, a possible fate of the solutions is to evolve to long-time traveling waves or to exhibit permanent chaotic behavior. Indeed, 
a weakly nonlinear analysis of Eq. (7) with $\bar{E}=0$ will result in the Kuramoto-Sivashinsky equation [78], which is a paradigmatic equation that exhibits chaos. As typical with Benney-type equations which include an inertial term proportional to $\partial_{x}\left(h^{6} \partial_{x} h\right)$, the solution may also blow up in finite time [79]. This blowup is due to a breakdown in the assumptions leading to the Benney equation and is not physical. In simulations the Reynolds number is kept sufficiently small to avoid this blowup. In this section we investigate the fates of the solutions of Eq. (7) depending on the values of the dimensionless parameters.

\section{Finite-time rupture: $K=0$}

When $K=0$ we observe that the generic behavior of solutions to Eq. (7) is to rupture $(h \rightarrow 0)$ at a finite point $x_{r}$ and finite time $t_{r}$. This rupture will correspond to a singularity of the solution, as the time and space derivatives will become infinite at the rupture point. The behavior close to rupture can be found by performing the similarity transformation

$$
h(x, t)=\left(t_{r}-t\right)^{\alpha} f(\xi, s), \quad \xi=\frac{x-x_{r}}{\left(t_{r}-t\right)^{\beta}}, s=-\ln \left(t_{r}-t\right) .
$$

Under this transformation we obtain $\partial_{t} h=\left(t_{r}-t\right)^{\alpha-1}\left(\partial_{s} f-\alpha f+\beta \xi \partial_{\xi} f\right)$ and each term in Eq. (7) is scaled by a power of $\left(t_{r}-t\right)$. The exponents $\alpha$ and $\beta$ are determined by balancing the time derivative term with surface tension [proportional to $\left(t_{r}-t\right)^{4 \alpha-4 \beta}$ ] and Marangoni or vapor recoil terms [proportional to $\left(t_{r}-t\right)^{\alpha-2 \beta}$ ]. Thus, we can write

$$
\alpha-1=4 \alpha-4 \beta=\alpha-2 \beta \Rightarrow \alpha=\frac{1}{3}, \quad \beta=\frac{1}{2} .
$$

Under this scaling, it can be easily checked that every other term in Eq. (7) is multiplied by a higher power of $\left(t_{r}-t\right)$ and is thus negligible in the limit that rupture is reached, $t \rightarrow t_{r}$. In particular, this rupture will occur independently of the presence of evaporative mass loss $(\bar{E}>0)$ and shear $(\tau>0)$.

Close to rupture, the scaled profile satisfies

$$
\partial_{s} f-\frac{1}{3} f+\frac{1}{2} \xi \partial_{\xi} f+\epsilon \partial_{\xi}\left[\left(\mathcal{M}+V_{r}\right) \partial_{\xi} f+\bar{S} f^{3} \partial_{\xi \xi \xi} f\right]=0
$$

where the neglected terms go to zero as $s \rightarrow \infty\left(t \rightarrow t_{r}\right)$. For instance, the evaporative mass loss term will be proportional to $\left(t_{r}-t\right)^{1-2 \alpha}=\left(t_{r}-t\right)^{1 / 3} \rightarrow 0$, while the shear stress term will be proportional to $\left(t_{r}-t\right)^{1+\alpha-\beta}=\left(t_{r}-t\right)^{5 / 6} \rightarrow 0$. The parameters $\epsilon, \mathcal{M}, V_{r}$, and $\bar{S}$ may be scaled out by taking

$$
\xi=\epsilon^{1 / 2}\left(\mathcal{M}+V_{r}\right)^{1 / 2} X, \quad f=\epsilon^{1 / 3}\left(\mathcal{M}+V_{r}\right)^{2 / 3} \bar{S}^{-1 / 3} F
$$

so that we obtain

$$
\partial_{s} F-\frac{1}{3} F+\frac{1}{2} X \partial_{X} F+\partial_{X}\left[\left(\partial_{X} F+F^{3} \partial_{X X X} F\right]=0 .\right.
$$

The thin-film equation corresponding to Eq. (23) is a special case of the family of thin-film-type equations with similarity solutions considered in Dallaston et al. [55]. In their work it was shown that Eq. (23) has an infinite number of steady states $\left(\partial_{s} F=0\right)$, corresponding to similarity solutions, the first of which is linearly stable. This situation is qualitatively similar to the rupture due to van der Waals attraction [76,77], and the connection between that problem and Eq. (23) was made in Ref. [55] via numerical continuation. The existence of a stable similarity solution implies that the rupture behavior of Eq. (7) is universal in the $K=0$ case.

We demonstrate the convergence to the universal rupture profile by solving Eq. (7) numerically until rupture and rescaling the interface data and spatial coordinate at each point in time using the transformations represented by Eqs. (20) and (22). Figure 15(a) shows the rescaled numerical interface of a film free of any gas shear $(\tau=0)$ at $t_{r}^{\prime}=t_{r}-t=2.596$, in comparison to the analytical self-similar solution to Eq. (23). The numerical and analytical profiles are divergent from each other, which is attributed to the fact that at this point in time the interface is still not close 

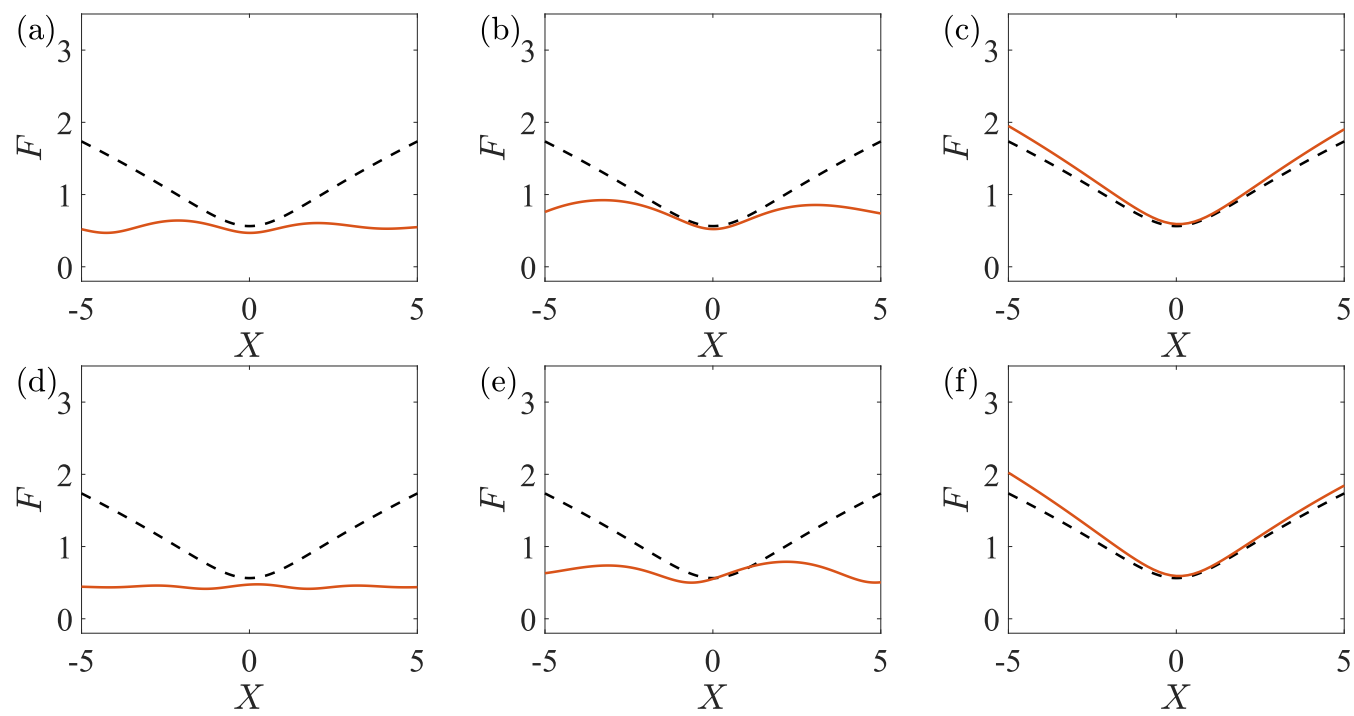

FIG. 15. Scaled numerical interface compared to the analytical self-similar solution of Eq. (23) at different times towards rupture for $K=0, \beta=0, \operatorname{Re}=1, \mathcal{M}=0.5, \bar{E}=0, V_{r}=1, t_{f}=0$, and (a)-(c) $\tau=0$ and (d)-(f) $\tau=0.3$ : (a) $t_{r}^{\prime}=2.596$, (b) $t_{r}^{\prime}=1.096$, (c) $t_{r}^{\prime}=0.01$, (d) $t_{r}^{\prime}=5.099$, (e) $t_{r}^{\prime}=1.599$, and (f) $t_{r}^{\prime}=0.01$. Note that $t_{r}^{\prime}=t_{r}-t$.

enough to rupture to satisfy the assumptions upon which Eq. (23) is based. However, as the value of $t_{r}^{\prime}$ decreases and $h \rightarrow 0$, the scaled numerical profile approaches and eventually agrees with the analytical one, as seen in Figs. 15(b) and 15(c), respectively.

Figures 15(d)-15(f) compare the numerical and analytical profiles at different values of $t_{r}^{\prime}$ for the same parameter set, but with an applied gas shear $(\tau=0.3)$, where the same dynamic is observed. Note that when the film is not close to rupture, the scaled numerical profiles corresponding to the cases with and without gas shear are dissimilar due to the effect of the nonzero shear stress being significant at these film thicknesses. However, as the film approaches rupture the role of the shear stress is severely diminished to the extent where it becomes negligible [see Figs. 15(c) and 15(f)]. This ultimately goes to show that the rupture mechanism for $K=0$ is indeed universal. Note that if $K$ is small but nonzero, the solution to Eq. (7) will initially tend to the stable similarity solution of Eq. (20) until $h$ and $K$ are comparable in size, after which a new dynamic regime appears. We demonstrate this numerically by computing the scaled numerical interface as the film approaches rupture for $K=0.03$ (see Fig. 16). As $h \rightarrow 0$, the scaled numerical solution approaches the analytical solution, as seen in Figs. 16(a)-16(c). However, as $h$ becomes smaller than $K$, the numerical solution departs from the analytical profile and exhibits new dynamic behavior [see Figs. 16(d)-16(f)].

\section{Nonsingular rupture: $K>0$ and $\bar{E}>0$}

The similarity solution discussed in Sec. IV D 1 is valid if $K=0$ or in the intermediate time when $\min (h) \gg K$. However, when $h \ll K$, the dynamics will be different and the behavior depends on whether the evaporative mass loss terms are present $(\bar{E}>0)$ or absent $(\bar{E}=0)$. When $\bar{E}>0$, the evaporative mass loss term cannot be neglected in any self-similar analysis. However, this term is not a destabilizing term and we do not see the universal self-similarity that results from the competition between surface tension and Marangoni or vapor recoil forces. Instead, the thickness goes to zero at a point with finite speed $\left(\partial_{t} h \sim-\bar{E} / K\right)$ without the appearance of any singularities in the solutions. This conclusion is corroborated numerically in Fig. 17, where it is seen that the scaled numerical 

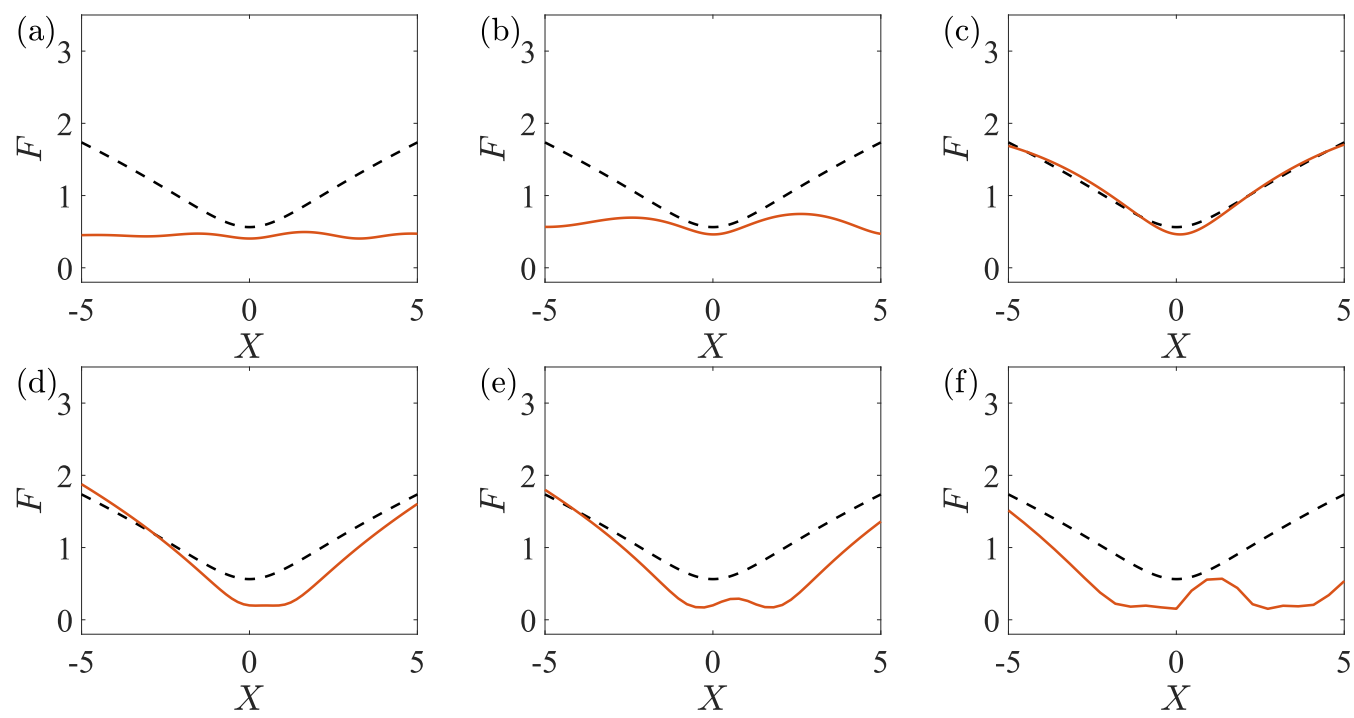

FIG. 16. Scaled numerical interface compared to the analytical self-similar solution at different times towards rupture for $K=0.03, \beta=0, \operatorname{Re}=1, \mathcal{M}=0.5, \bar{E}=0, V_{r}=1, \tau=0$, and $t_{f}=0$ : (a) $t_{r}^{\prime}=4.939$, (b) $t_{r}^{\prime}=1.939$, (c) $t_{r}^{\prime}=0.121$, (d) $t_{r}^{\prime}=0.007$, (e) $t_{r}^{\prime}=0.003$, and (f) $t_{r}^{\prime}=0.001$. Observe the deviation from the analytical self-similar solution in plots (d)-(f) and the appearance of the new dynamic behavior. Note that $t_{r}^{\prime}=t_{r}-t$.

interface does not go towards the self-similar solution but instead flattens out, indicating nonsingular rupture.

\section{Infinite-time rupture: $K>0, \bar{E}=0$, and $\tau \geqslant 0$}

If $K>0$ and $\bar{E}=\tau=0$, we find the most important terms again come from balancing the time derivative with the Marangoni force and the surface tension. However, due to the effect of $K$ on the Marangoni term, we obtain the approximate behavior

$$
\partial h_{t}+\epsilon \partial_{x}\left[\frac{\mathcal{M}}{K^{2}} h^{2} \partial_{x} h+\bar{S} h^{3} \partial_{x x x} h\right] .
$$

For this equation an infinite-time, rather than finite-time, similarity ansatz is appropriate:

$$
h(x, t)=t^{-\alpha} f(\xi, s), \quad \xi=t^{\beta}\left(x-x_{0}\right), s=\ln t .
$$
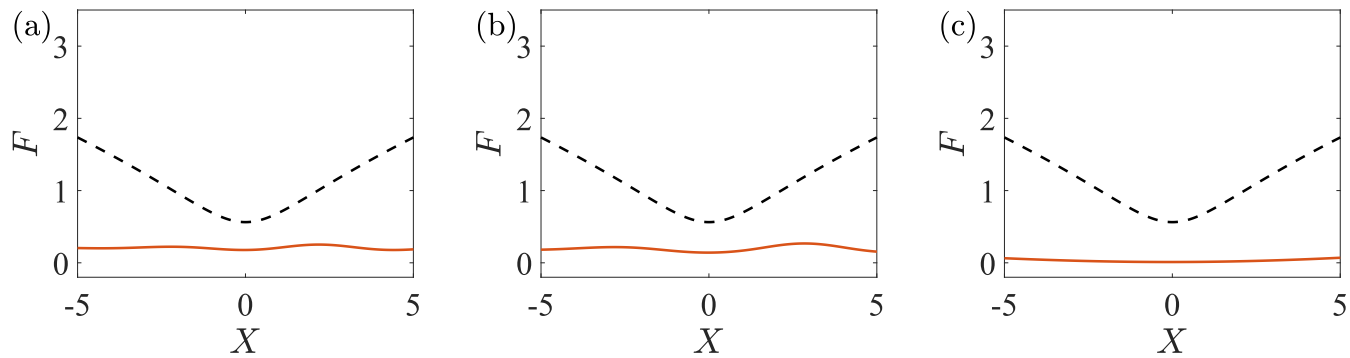

FIG. 17. Scaled numerical interface compared to the analytical self-similar solution at different times towards rupture for $K=0.2, \beta=0, \operatorname{Re}=1, \mathcal{M}=0.5, \bar{E}=0.05, V_{r}=1, \tau=0$, and $t_{f}=0$ : (a) $t_{r}^{\prime}=1.829$, (b) $t_{r}^{\prime}=1.079$, and (c) $t_{r}^{\prime}=0.014$. Note that $t_{r}^{\prime}=t_{r}-t$. 

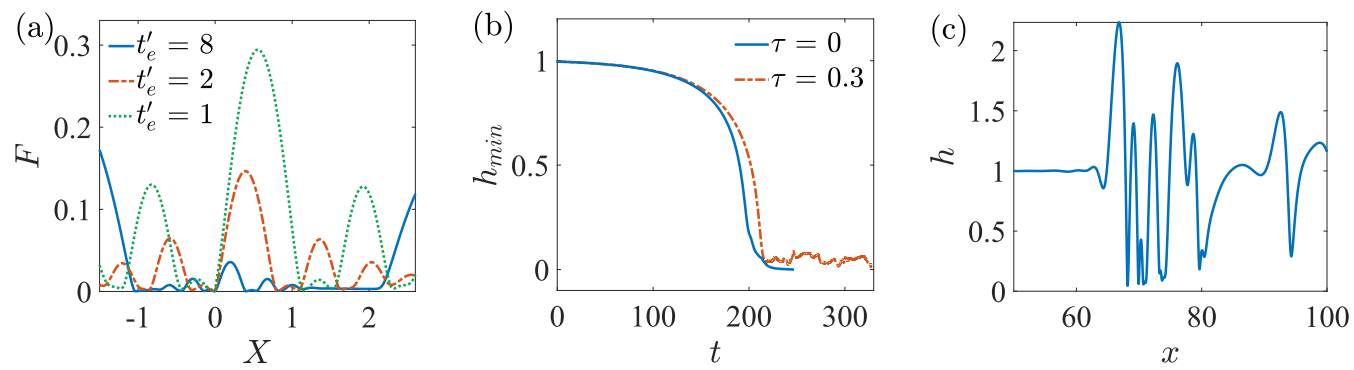

FIG. 18. Film evolution close to rupture for $K=0.4, \beta=0, \operatorname{Re}=1, \mathcal{M}=0.5, \bar{E}=0, V_{r}=1$, and $t_{f}=0$. (a) Scaled numerical interface for $\tau=0$ at different values of $t_{e}^{\prime}=t_{\text {end }}-t$. (b) Minimum interface height versus time for $\tau=0$ and $\tau=0.3$. (c) Chaotic interface evolution for $\tau=0.3$ at $t=286.1$.

Balancing the three terms gives $\alpha=1$ and $\beta=1 / 2$, resulting in the equation

$$
\partial_{s} f-f+\frac{1}{2} \xi \partial_{\xi} f+\epsilon \partial_{\xi}\left[\frac{\mathcal{M}}{K^{2}} f^{2} \partial_{\xi} f+\bar{S} f^{3} \partial_{\xi \xi \xi} f\right]=0 .
$$

All other terms in Eq. (7) are of lower power in $t$ and therefore negligible in the limit $t \rightarrow \infty$. As previously observed in Refs. [52,53], the behavior of Eq. (24), as time becomes large, is to form an infinite cascade of geometrically shrinking oscillations as $h \rightarrow 0$. This likely corresponds to the existence of a periodic (in $s$ ) solution to Eq. (26) for this phenomenon observed in finite-time rupture [55].

On the other hand, if $K>0$ and $\bar{E}=0$ but $\tau>0$, the nonzero shear stress has the effect of removing the infinite-time rupture solution. If we carry out the similarity transformation as in Eq. (26) but have nonzero shear, we obtain

$$
\partial_{s} f-f+\frac{1}{2} \xi \partial_{\xi} f+\epsilon \partial_{\xi}\left[\frac{\mathcal{M}}{K^{2}} f^{2} \partial_{\xi} f+\bar{S} f^{3} \partial_{\xi \xi \xi} f\right]+t^{1 / 2} \partial_{\xi}\left[\frac{\tau}{2} f^{2}\right]=0 .
$$

The positive power of $t$ on the shear term implies that the cascading similarity solution cannot be reached. In this case, it is observed that the solutions evolve chaotically as the film becomes very thin.

Figure 18(a) shows the scaled numerical interface at different times towards rupture. As $h \rightarrow 0$, the scaled numerical interface's oscillations form a geometric hierarchy which repeats for a vanishing spatial coordinate. Since the film in this case ruptures in infinite time, note the usage of $t_{e}^{\prime}=t_{\text {end }}-t$ instead of $t_{r}^{\prime}$, where $t_{\text {end }}$ is the value of time at which the numerical solution stopped. Figure 18(b) plots the minimum interface height versus time for $\tau=0$ and $\tau=0.3$. It is observed that in the case of $\tau=0, h_{\min }$ approaches zero asymptotically, which is consistent with infinite-time rupture. However, for nonzero shear $(\tau=0.3), h_{\min }$ behaves erratically when it becomes small. This chaotic interface evolution is further highlighted by plotting the fluid interface in this regime (i.e., $t=286.1$ ), as shown in Fig. 18(c).

\section{CONCLUSION}

We have studied the effects of a shearing gas on the evolution of an evaporating liquid film via linear stability theory, numerical simulation, and self-similarity analysis. The one-sided model of Burelbach et al. [20] was used to account for the evaporation of the fluid film, while the shear stress imparted by the gas on the free interface was prescribed as a constant quantity, denoted by $\tau$, whose role was confined to modifying the base flow. The main difference of our work lies in investigating the effects of a shearing gas on the temporal and spatiotemporal stability of perturbations caused by thermal effects including thermocapillary and evaporation forces. 
Through a linear stability analysis we found that (i) the shear gas's influence is much more pronounced on the temporal growth rate of the inertial $H$-mode instability and (ii) its influence depends on whether the fluid film under consideration is moving $(\beta \neq 0)$ or stationary $(\beta=0)$. In a moving film the shear stress modifies the flow's existing inertia and therefore affects both the temporal growth rate and the perturbation phase speed, while in a stationary film it only changes the perturbation phase speed. We found that for a sufficiently negative value of $\tau$ the $H$-mode instability is completely suppressed. This result qualitatively matched that found by Lavalle et al. [38], who used a more complex model (limited to isothermal films), combined with experimental validation. We also investigated how the evaporation and consequent thinning of the film affects the perturbations and their relationship with the shearing gas. We found that the thinning of the film in general suppresses the $H$-mode instability, but enhances the thermal instability mode. The influence of the shearing gas on both these instability modes is diminished as the film thins due to the increasing dominance of viscous forces. Additionally, the thinning of the film results in a reduction in perturbation phase speed, indicating a resistance for the convection of the perturbations and possibly a transition into the absolute instability regime. Our subsequent spatiotemporal analysis confirmed this result, showing that the ability of the base flow to advect the perturbations away from their origin is diminished as the film thins. However, we found that the thinning of the film results in the activation of new modes with higher group velocities, resulting in an overall faster spread of the perturbations via their own mechanism.

The interface evolution equation was solved numerically to study the film's evolution when subject to finite perturbations. A set of dimensionless parameters was chosen to show the evolution of the flow dynamics of an evaporating film from stable to convectively unstable and ultimately to absolutely unstable. A temporal analysis showed that the dynamic behavior observed in the linear regime persists qualitatively for finite perturbations. Moreover, a subsequent spatiotemporal investigation demonstrated the effects of the film thinning due to evaporation on its evolution, where it was shown to cause a transition in the type of instability from convective to absolute. Additionally, the impulse response wave packets were computed numerically for finite perturbations where we found that, in the case of weaker thermal instabilities, the linear and nonlinear responses are different. In particular, the nonlinear absolute growth rate is higher than its linear counterpart, indicating that finite perturbations are relatively less convective than infinitesimal disturbances. Consequently, according to Dee and Langer's [75] criterion, the front velocities of nonlinear instabilities are nonlinearly selected as they are imposed by the nonlinearly saturated wave packet. On the other hand, when the thermal mode instability is relatively strong the linear and nonlinear wave fronts converge, suggesting that the wave-front dynamics are dictated by infinitesimal modes governed by the linear dispersion relationship.

Finally, we investigated the role of the dimensionless shear stress, among other dimensionless parameters, on the rupture mechanism by means of a self-similarity analysis, which was compared to the scaled numerical simulation data. Our results showed that the dominant parameters in determining the fates of the solutions to the evolution equation are the equilibrium parameter $K$, the evaporation number $\bar{E}$, and the constant shear stress $\tau$. In the case of $K=0$ the film ruptures in finite time where this rupture mechanism is universal. When $K$ is nonzero but small the solutions to the evolution equation will approach the stable self-similarity solution until $h$ and $K$ are comparable in size, beyond which a new dynamic behavior is detected. On the other hand, when $K>0$ and $\bar{E}>0$ the film thickness goes to zero at a point with finite speed $\partial_{t} h \sim-\bar{E} / K$ without the appearance of any singularities in the solutions. Again we find this rupture mechanism to be universal regardless of the value of $\tau$. Furthermore, in the case of $K>0$ and $\bar{E}=0$, the film ruptures in infinite time, where our analysis identified the appearance of an infinite cascade of geometrically shrinking oscillations when $\tau=0$ and chaotic evolution when $\tau>0$.

The introduction of a shearing gas into the problem of an evaporating thin liquid film adds a great deal of complexity and richness to an already interesting problem, which has ample room for further investigation beyond our present work. Due to the limitations of the Benney equation, improved models could be used to capture the interface evolution with a higher degree of accuracy 
and for higher Reynolds numbers such as the models utilizing the weighted-residual methods developed by Ruyer-Quil and Manneville [12,13]. Additionally, the small wave number limit could be overcome by introducing the shearing gas effects into the Orr-Sommerfeld eigenvalue problem for an evaporating liquid film, as done by Mohamed and Biancofiore [22]. The results obtained from these improved models could be then compared to those obtained via the direct numerical simulation of the two-phase problem, via ad hoc algorithms developed for evaporating flows [80].

Moreover, the limitations of our shearing gas model could be overcome by considering the full interaction between the shearing gas and the liquid film using more advanced models such as those formulated by O'Náraigh et al. [48] and Vellingiri et al. [43]. These formulations would allow including the role of the normal stress imparted by the gas on the film's surface. In addition, it would be possible also to focus specifically on the interaction between a turbulent gas and the thermal instabilities. For instance, the three-dimensional effects of the turbulent gas could have a significant effect on other instability modes such as the transverse $P$ mode [19]. These enhanced models would also allow studying the role of practically relevant effects such as film confinement.

\section{ACKNOWLEDGMENTS}

L.B. and O.A.A.M. would like to acknowledge the Turkish National Research Agency (TÜBITAK) for supporting this work through Project No. 216M001. L.B. and M.C.D. would like to thank TÜBITAK for the 2221 Visiting Fellowship (Grant No. 1059B211800297) through which this work had significantly gained momentum.

[1] C. Marangoni, T. Peruzzo, I. G. B. Parisotto, V. W. Ricardo, C. A. Claumann, K. W. Milanez, M. B. H. Mantelli, M. B. Quadri, A. Bolzan, M. C. dos Santos, L. C. Medina, and R. A. F. Machado, Falling film distillation column with heat transfer by means of a vapor chamber. Part II: Operation with a temperature profile, Chem. Eng. Commun. 206, 1006 (2019).

[2] A. B. Erasmus and I. Nieuwoudt, Mass transfer in structured packing: A wetted-wall study, Ind. Eng. Chem. Res. 40, 2310 (2001).

[3] A. L. Kohl and R. Nielsen, Gas Purification (Gulf, Houston, 1997).

[4] A. H. Lefebvre and V. G. McDonell, Atomization and Sprays (Hemisphere, New York, 1989).

[5] G. A. Lawrence, F. K. Browand, and L. G. Redekopp, The stability of a sheared density interface, Phys. Fluids A 3, 2360 (1991).

[6] D. Lucas, C. P. Caulfield, and R. R. Kerswell, Layer formation in horizontally forced stratified turbulence: Connecting exact coherent structures to linear instabilities, J. Fluid Mech. 832, 409 (2017).

[7] P. L. Kapitza, Wave flow of thin layers of a viscous fluid: I. Free flow, J. Exp. Theor. Phys. 18, 3 (1948).

[8] P. L. Kapitza and S. P. Kapitza, Wave flow of thin layers of a viscous fluid: III. Experimental study of undulatory flow conditions, J. Exp. Theor. Phys. 19, 105 (1949).

[9] T. B. Benjamin, Wave formation in laminar flow down an inclined plane, J. Fluid Mech. 2, 554 (1957).

[10] C.-S. Yih, Stability of liquid flow down an inclined plane, Phys. Fluids 6, 321 (1963).

[11] D. J. Benney, Long waves on liquid films, J. Math. Phys. 45, 150 (1966).

[12] C. Ruyer-Quil and P. Manneville, Improved modeling of flows down inclined planes, Eur. Phys. J. B 15, 357 (2000).

[13] C. Ruyer-Quil and P. Manneville, Further accuracy and convergence results on the modeling of flows down inclined planes by weighted-residual approximations, Phys. Fluids 14, 170 (2002).

[14] M. K. Smith, The mechanism for the long-wave instability in thin liquid films, J. Fluid Mech. 217, 469 (1990).

[15] S. Kalliadasis, C. Ruyer-Quil, B. Scheid, and M. G. Velarde, Falling Liquid Films (Springer, London, 2012). 
[16] G. Dietze, On the Kapitza instability and the generation of capillary waves, J. Fluid Mech. 789, 368 (2016).

[17] S. Sreenivasan and S. P. Lin, Surface tension driven instability of a liquid film flow down a heated incline, Int. J. Heat Mass Transfer 21, 1517 (1978).

[18] R. E. Kelly, S. H. Davis, and D. A. Goussis, in Proceedings of the Eighth International Heat Transfer Conference, San Francisco, 1986, edited by C. L. Tien, V. P. Carey, and J. K. Ferrell (Hemisphere, New York, 1986), p. 1937.

[19] D. A. Goussis and R. E. Kelly, Surface wave and thermocapillary instabilities in a liquid film flow, J. Fluid Mech. 223, 25 (1991).

[20] J. P. Burelbach, S. G. Bankoff, and S. H. Davis, Nonlinear stability of evaporating/condensing liquid films, J. Fluid Mech. 195, 463 (1988).

[21] S. W. Joo, S. H. Davis, and S. G. Bankoff, Long-wave instabilities of heated falling films: Twodimensional theory of uniform layers, J. Fluid Mech. 230, 117 (1991).

[22] H. Mohamed and L. Biancofiore, Linear stability analysis of evaporating falling liquid films, Int. J. Multiphase Flow 130, 103354 (2020).

[23] V. S. Ajaev and G. M. Homsy, Steady vapor bubbles in rectangular microchannels, J. Colloid Interface Sci., 240, 259 (2001).

[24] V. S. Ajaev, Spreading of thin volatile liquid droplets on uniformly heated surfaces, J. Fluid Mech. 528, 279 (2005).

[25] V. S. Ajaev, Evolution of dry patches in evaporating liquid films, Phys. Rev. E 72, 031605 (2005).

[26] H. Ji and T. P. Witelski, Instability and dynamics of volatile thin films, Phys. Rev. Fluids 3, 024001 (2018).

[27] S. D. Howison, J. A. Moriarty, J. R. Ockendon, E. L. Terrill, and S. K. Wilson, A mathematical model for drying paint layers, J. Eng. Math. 32, 377 (1997).

[28] O. E. Shklyaev and E. Fried, Stability of an evaporating thin liquid film, J. Fluid Mech. 584, 157 (2007).

[29] E. Fried, Sharp-interface nematic-isotropic phase transformations with flow, Arch. Ration. Mech. Anal. 190, 227 (2008).

[30] R. D. Deegan, O. Bakajin, T. F. Dupont, G. Huber, S. R. Nagel, and T. A. Witten, Capillary flow as the cause of ring stains from dried liquid drops, Nature (London) 389, 827 (1997).

[31] R. D. Deegan, O. Bakajin, T. F. Dupont, G. Huber, S. R. Nagel, and T. A. Witten, Contact line deposits in an evaporating drop, Phys. Rev. E 62, 756 (2000).

[32] E. Sultan, A. Boudaoud, and M. B. Amar, Diffusion-limited evaporation of thin polar liquid films, J. Eng. Math. 50, 209 (2004).

[33] E. Sultan, A. Boudaoud, and M. B. Amar, Evaporation of a thin film: Diffusion of the vapour and Marangoni instabilities, J. Fluid Mech. 543, 183 (2005).

[34] T. J. Hanratty and J. M. Engen, Interaction between a turbulent air stream and a moving water surface, AIChE J. 3, 299 (1957).

[35] A. D. D. Craik, Wind-generated waves in thin liquid films, J. Fluid Mech. 26, 369 (1966).

[36] L. O'Náraigh, P. D. M. Spelt, and T. A. Zaki, Turbulent flow over a liquid layer revisited: Multi-equation turbulence modelling, J. Fluid Mech. 683, 357 (2011).

[37] G. F. Dietze and C. Ruyer-Quil, Wavy liquid films in interaction with a confined laminar gas flow, J. Fluid Mech. 722, 348 (2013).

[38] G. Lavalle, Y. Li, S. Mergui, N. Grenier, and G. F. Dietze, Suppression of the Kapitza instability in confined falling liquid films, J. Fluid Mech. 860, 608 (2019).

[39] A. C. King, E. O. Tuck, and J. M. Vanden-Broeck, Air-blown waves on thin viscous sheets, Phys. Fluids A 5, 973 (1993).

[40] M. Sivapuratharasu, S. Hibberd, M. E. Hubbard, and H. Power, Inertial effects on thin-film wave structures with imposed surface shear on an inclined plane, Physica D 325, 86 (2016).

[41] C. J. Shearer and J. F. Davidson, The investigation of a standing wave due to gas blowing upwards over a liquid film; its relation to flooding in wetted-wall columns, J. Fluid Mech. 22, 321 (1965).

[42] A. G. Cetinbudaklar and G. J. Jameson, The mechanism of flooding in vertical countercurrent two-phase flow, Chem. Eng. Sci. 24, 1669 (1969). 
[43] R. Vellingiri, D. Tseluiko, and S. Kalliadasis, Absolute and convective instabilities in counter-current gas-liquid film flows, J. Fluid Mech. 763, 166 (2015).

[44] R. J. Briggs, Electron-Stream Interaction with Plasmas (MIT Press, Cambridge, 1964).

[45] P. Huerre and P. A. Monkewitz, Local and global instabilities in spatially developing flows, Annu. Rev. Fluid Mech. 22, 473 (1990).

[46] L. Brevdo, P. Laure, F. Dias, and T. J. Bridges, Linear pulse structure and signalling in a film flow on an inclined plane, J. Fluid Mech. 396, 37 (1999).

[47] S. W. Joo and S. H. Davis, Instabilities of three-dimensional viscous falling films, J. Fluid Mech. 242, 529 (1992).

[48] L. O’Náraigh, P. D. M. Spelt, and S. J. Shaw, Absolute linear instability in laminar and turbulent gas-liquid two-layer channel flow, J. Fluid Mech. 714, 58 (2013).

[49] D. Tseluiko and S. Kalliadasis, Nonlinear waves in counter-current gas-liquid film flow, J. Fluid Mech. 673, 19 (2011).

[50] M. B. Williams and S. H. Davis, Nonlinear theory of film rupture, J. Colloid Interface Sci. 90, 220 (1982).

[51] S. Krishnamoorthy, B. Ramaswamy, and S. W. Joo, Spontaneous rupture of thin liquid films due to thermocapillarity: A full-scale direct numerical simulation, Phys. Fluids 7, 2291 (1995).

[52] W. Boos and A. Thess, Cascade of structures in long-wavelength Marangoni instability, Phys. Fluids 11, 1484 (1999).

[53] S. Shklyaev, A. V. Straube, and A. Pikovsky, Superexponential droplet fractalization as a hierarchical formation of dissipative compactons, Phys. Rev. E 82, 020601(R) (2010).

[54] T. Wei and F. Duan, Interfacial stability and self-similar rupture of evaporating liquid layers under vapor recoil, Phys. Fluids 28, 124106 (2016).

[55] M. C. Dallaston, M. A. Fontelos, D. Tseluiko, and S. Kalliadasis, Discrete Self-Similarity in Interfacial Hydrodynamics and the Formation of Iterated Structures, Phys. Rev. Lett. 120, 034505 (2018).

[56] H. Ji and T. P. Witelski, Finite-time thin film rupture driven by modified evaporative loss, Physica D 342, 1 (2017).

[57] J. M. Delhaye, Jump conditions and entropy sources in two-phase systems. Local instant formulation, Int. J. Multiphase Flow 1, 395 (1974).

[58] H. J. Palmer, The hydrodynamic stability of rapidly evaporating liquids at reduced pressure, J. Fluid Mech. 75, 487 (1976).

[59] T. F. Lin, C. J. Chang, and W. M. Yan, Analysis of combined buoyancy effects of thermal and mass diffusion on laminar forced convection heat transfer in a vertical tube, J. Heat Transfer 110, 337 (1988).

[60] W. M. Yan and C. Y. Soong, Convective heat and mass transfer along an inclined heated plate with film evaporation, Int. J. Heat Mass Transfer 38, 1261 (1995).

[61] H. Ding and P. D. M. Spelt, Onset of motion of a three-dimensional droplet on a wall in shear flow at moderate Reynolds numbers, J. Fluid Mech. 599, 341 (2008).

[62] H. Ding, M. N. H. Gilani, and P. D. M. Spelt, Sliding, pinch-off and detachment of a droplet on a wall in shear flow, J. Fluid Mech. 644, 217 (2010).

[63] C. Paterson, S. K. Wilson, and B. R. Duffy, Rivulet flow round a horizontal cylinder subject to a uniform surface shear stress, Q. J. Mech. Appl. Math. 67, 567 (2014).

[64] J. M. Sullivan, C. Paterson, S. K. Wilson, and B. R. Duffy, A thin rivulet or ridge subject to a uniform transverse shear stress at its free surface due to an external airflow, Phys. Fluids 24, 082109 (2012).

[65] J. M. Sullivan, S. K. Wilson, and B. R. Duffy, A thin rivulet of perfectly wetting fluid subject to a longitudinal surface shear stress, Q. J. Mech. Appl. Math. 61, 25 (2008).

[66] S. K. Wilson, J. M. Sullivan, and B. R. Duffy, The energetics of the breakup of a sheet and of a rivulet on a vertical substrate in the presence of a uniform surface shear stress, J. Fluid Mech. 674, 281 (2011).

[67] S. Davis, in Waves on Fluid Interfaces, edited by R. E. Meyer (Academic, Cambridge, 1983), p. 291.

[68] S. H. Davis, The stability of time-periodic flows, Annu. Rev. Fluid Mech. 8, 57 (1976).

[69] L. Biancofiore and F. Gallaire, Counterpropagating Rossby waves in confined plane wakes, Phys. Fluids 24, 074102 (2012).

[70] L. Biancofiore, F. Gallaire, and E. Heifetz, Interaction between counterpropagating Rossby waves and capillary waves in planar shear flows, Phys. Fluids 27, 044104 (2015). 
[71] G. K. Batchelor, H. K. Moffatt, and M. G. Worster, Perspectives in Fluid Dynamics: A Collective Introduction to Current Research (Cambridge University Press, Cambridge, 2002).

[72] MATLAB, Version 9.9.0.1592791 (R2020b), Update 5 (Mathworks, Inc., Natick, 2020).

[73] P. Huerre and P. A. Monkewitz, Absolute and convective instabilities in free shear layers, J. Fluid Mech. 159, 151 (1985).

[74] I. Delbende and J.-M. Chomaz, Nonlinear convective/absolute instabilities in parallel two-dimensional wakes, Phys. Fluids 10, 2724 (1998).

[75] G. Dee and J. S. Langer, Propagating Pattern Selection, Phys. Rev. Lett. 50, 383 (1983).

[76] T. P. Witelski and A. J. Bernoff, Stability of self-similar solutions for van der Waals driven thin film rupture, Phys. Fluids 11, 2443 (1999).

[77] W. W. Zhang and J. R. Lister, Similarity solutions for van der Waals rupture of a thin film on a solid substrate, Phys. Fluids 11, 2454 (1999).

[78] G. I. Sivashinsky and D. M. Michelson, On irregular wavy flow of a liquid film down a vertical plane, Prog. Theor. Phys. 63, 2112 (1980).

[79] A. Pumir, P. Manneville, and Y. Pomeau, On solitary waves running down an inclined plane, J. Fluid Mech. 135, 27 (1983).

[80] M. Irfan and M. Muradoglu, A front tracking method for direct numerical simulation of evaporation process in a multiphase system, J. Comput. Phys. 337, 132 (2017). 